\title{
Cambios de la Tasa de Política y su Efecto en la Estructura a Plazo de Colombia*
}

\author{
Luis EdUARDo ARANGO \\ Banco de la República, Colombia \\ ANDRÉs GONZÁLEZ \\ Banco de la República, Colombia \\ JOHN JAIRO LEÓN \\ Banco de la República, Colombia \\ Luis Fernando Melo \\ Banco de la República, Colombia
}

This paper analyzes the effects of changes of Banco de la República's policy interest rate in the term structure. The evidence suggests that, in daily frequency, these reactions are not significant. However, with weekly data we found an anticipated reaction (one and three weeks before the movement) in the term structure in response to a change in the policy interest rate. Unfortunately the reaction has a "steepness effect" on the term structure, which may not support the expectations hypothesis of interest rates or may suggest a weak credibility and transparency of Banco de la República or both.

\section{JEL: E43, E52, F31}

Keywords: Tasas de Política, Estructura a Plazo de Tasas de Interés, Hipótesis de Paridad Descubierta, Transparencia, Credibilidad.

\section{INTRODUCCIÓN}

El esquema de metas de inflación seguido por algunos países se apoya en el supuesto crucial de que la autoridad monetaria ejerce una influencia importante

\footnotetext{
* Los puntos de vista expresados en este documento corresponden estrictamente a los autores y no necesariamente reflejan los del Banco de la República o su Junta Directiva. Se agradece a Carolina Gómez, Felipe Lega, Juliana Mera y Mauricio Ruiz tanto por el suministro de la información sobre tasas de interés como por sus explicaciones. De igual forma, se agradecen las preguntas, comentarios y sugerencias de Fernando Arias, Rocío Betancourt, Luz Adriana Flórez, Munir Jalil, Carlos Esteban Posada, Hernando Vargas y Daniel Velandia.

Email: larangth@banrep.gov.co
} 
sobre el conjunto de tasas de interés (activas y pasivas) de la economía, a través de los movimientos de la tasa referencial que dicta la regla de política ${ }^{1}$; dicho conjunto incluye la tasa interbancaria, la de los créditos de consumo, certificados de depósito a término, hipotecas, etc. De manera breve, suponiendo que las tasas de interés tienen un componente asociado a la tasa de interés real y otro a las expectativas de inflación, se puede explicar que, mediante los movimientos de las tasas de política, la autoridad monetaria pueda afectar la trayectoria de las expectativas de inflación futura y, a través de éstas, las tasas de interés asociadas a mayores plazos.

En principio, descifrar el contenido informativo de la estructura a plazo suele ser una tarea difícil; sin embargo, existe algún acuerdo sobre el mismo. Por ejemplo, se dice que el tramo corto de la estructura a plazo, que en este trabajo interpretamos como la representación gráfica de la estructura a plazo, refleja las acciones de la autoridad monetaria y, en alguna medida, las condiciones reales y financieras de la economía. Así, una economía vigorosa y con presiones inflacionarias tendrá un tramo corto elevado. El tramo medio de la curva refleja las expectativas de inflación en el mediano plazo y, por lo tanto, los objetivos de la autoridad monetaria, los cambios esperados en su postura y las tasas de retorno reales son explicadas por el ciclo o por cambios en la tendencia de crecimiento. El tramo largo es influenciado por las percepciones de crecimiento potencial de la economía, las expectativas de inflación de largo plazo y la credibilidad del banco central en su lucha contra la inflación (véase Fernández, 2000 y Arango y Flórez, 2008 y las referencias citadas allí, e.g. BIS, 2005).

La hipótesis de que las intervenciones de la autoridad monetaria afectan las tasas de interés del mercado ha logrado verificarse con éxito en algunos países avanzados (Cook y Hahn, 1989; Dale, 1993; Roley y Sellon, 1995; Kuttner, 2001; y Demiralp y Jorda, 2004, entre otros). En general, los autores señalan que la reacción que tienen las tasas de los títulos, ante movimientos de las tasas de política por parte de la autoridad monetaria, se explica por cambios en las expectativas sobre los niveles de las tasas de política a lo largo de la vida de dichos títulos. Haldane y Read (1999) señalan que las respuestas de las tasas de interés a las intervenciones son una medida de los grados de transparencia y credibilidad de un régimen monetario. Este argumento será discutido más adelante.

En Colombia, el paso (pass-through) de las tasas de política del Banco de la República (BR) a las tasas de mercado ha sido una preocupación constante (véanse, por ejemplo, Julio 2001; Huertas, Jalil, Olarte y Romero, 2005; Amaya, 2006; Melo y Becerra, 2006; y Betancourt, Vargas y Rodríguez, 2006). No obstante, los trabajos realizados hasta ahora no han estudiado, bien sea por razones

${ }^{1}$ El Bank of England Quarterly Bulletin (1999, págs. 161-170) sugiere que las tasas oficiales afectan, además de las tasas de interés de mercado, los precios de los activos, las expectativas de inflación y el tipo de cambio, todas ellas con efectos tanto en la demanda doméstica como en la demanda externa neta. Mishkin (1995) da por sentada la influencia de la política monetaria en la tasa de interés y, con base en ello, presenta un argumento conocido como el "canal de tasa de interés" por medio del cual la política monetaria afecta la actividad económica. 
de interés empírico o de su aproximación teórica, dos aspectos importantes. En primer lugar, no hacen ninguna consideración sobre las expectativas de los agentes; $\mathrm{y}$, en segundo lugar, no incluyen en el conjunto de las tasas de mercado analizadas las de los títulos de endeudamiento negociados en el mercado secundario que, para el caso colombiano, constituyen las tasas de largo plazo y son por tanto los determinantes, según la teoría, de las decisiones de consumo e inversión.

Este trabajo tiene como propósito utilizar un enfoque que permita complementar las contribuciones anteriores. Más exactamente, este documento tiene como objetivo estimar el impacto que tienen los movimientos de las tasas de política del BR en la estructura a plazo de tasas de interés. Como representativas de ésta se utilizan las tasas de interés corriente (spot) y a plazo (forward) de la curva cero cupón en frecuencias diaria y semanal a diferentes plazos. El estudio comprende el período entre el 2 enero de 2002 y el 31 de enero de 2007. Se trata en total de 1.243 observaciones diarias o 266 semanales. Este período muestral tiene su justificación porque desde el 2 de enero de 2002 se tiene la posibilidad de construir la curva cero cupón en frecuencia diaria sin mayores interrupciones; algunos datos faltantes fueron estimados utilizando el método TRAMO de interpolación (véase Anexo 1) ${ }^{2}$.

¿Por qué es importante verificar el impacto de las tasas de política del BR en las tasas del mercado secundario de deuda? Las respuestas son, al menos, dos. En primer lugar, porque se daría una señal en favor del uso del esquema de metas de inflación; el BR estaría siendo exitoso al ser un factor determinante en la formación de expectativas sobre inflación futura. En segundo término, porque al verificarse la capacidad de afectación de las tasas de política a la estructura a plazo se estaría presentando la posibilidad de que operen al menos dos canales de transmisión de la política monetaria: el canal de tasa de interés y el de precios de otros activos. El primero de ellos entra en vigencia en la medida en que se altera el precio relativo del consumo presente en términos del consumo futuro y tanto las trayectorias de consumo como de inversión se ven modificadas. El segundo entra en vigor al modificarse el nivel de riqueza de los agentes (cambia el valor de sus activos) dado el uso que tiene la estructura a plazo ${ }^{3}$. Como la modificación en el precio de los activos no es, necesariamente, uniforme surgen posibilidades de inversión para los agentes ${ }^{4}$.

El enfoque de este trabajo difiere, en varios aspectos, del tradicional asociado a Cook y Hahn (1989), mediante el cual se observan las reacciones directas en las tasas de mercado por movimientos contemporáneos o con adelantos y rezagos de uno o dos días en las tasas de expansión en ecuaciones lineales de dos variables.

\footnotetext{
${ }^{2}$ En total se generaron datos para 14 días en los que no fue posible construir la curva por el escaso número de transacciones en el mercado secundario de deuda.

${ }^{3}$ Recordemos que las tasas de interés de la estructura a plazo se utilizan para valorar activos; por lo tanto, si estas se mueven al producirse modificaciones en las tasas oficiales del BR se estará modificando el nivel de riqueza de los agentes. Lo anterior no quiere decir, sin embargo, que el BR esté en capacidad de afectar la tasa de interés real de largo plazo de la economía.

${ }^{4}$ La verificación del paso de la tasa de política a las tasas de mercado habilita la justificación de otros canales de transmisión de la política monetaria como son los de crédito y tipo de cambio.
} 
Como se verá más adelante la estructura a plazo tiene un comportamiento que no parece estar ligado -al menos exclusivamente- al de las tasas de política del BR. El movimiento diario de la estructura a plazo sugiere la necesidad de controlar por otras variables. Por ello, en primer lugar, nuestro trabajo pregunta qué ocurre con la estructura a plazo los días en que no hay intervención por parte de la autoridad monetaria: ¿se queda, igualmente, quieta? Si esto no así, entonces, qué hace que la curva se mueva durante los días en los que el BR no modifica las tasas oficiales. En segundo término, el trabajo tiene en cuenta el carácter de economía pequeña y abierta que tiene Colombia. Por tal razón, además de considerar las variaciones en las tasas de política, utiliza una relación de paridad descubierta de intereses con la economía de Estados Unidos, nuestro principal referente financiero.

El trabajo se desarrolla de la siguiente manera. La primera sección es esta introducción. La segunda presenta la evolución de las variables básicas como la tasa de política y la estructura a plazo. La tercera sección discute los conceptos básicos de transparencia, credibilidad e hipótesis de expectativas. La cuarta comenta algunas contribuciones previas y presenta los primeros resultados siguiendo el enfoque de Cook y Hahn (1989). La quinta sección analiza el modelo utilizado para verificar la hipótesis de este trabajo. La sección sexta señala el enfoque econométrico adoptado, los datos y examina los resultados. La séptima sección concluye.

\section{Hechos BÁsICOS}

La Figura 1 presenta la trayectoria de la tasa de subasta de expansión, que actualmente es el principal instrumento de política del BR en el mercado de dinero. Dicha tasa materializa el precio al cual el BR ofrece un monto limitado de liquidez al mercado a través de un mecanismo de subasta. De acuerdo con la Figura 1, entre agosto de 1999 y junio de 2002 se observaron reducciones en dicha tasa, es decir, durante ese período el BR señaló caídas en el precio de la liquidez. Esto se sigue de un breve período de aumentos y disminuciones, pero desde mayo de 2006 se observan aumentos.

La Figura 2 muestra la magnitud de las intervenciones. En general, estas han sido de 100, 50 y 25 puntos básicos $(p b)$, con excepción del movimiento de noviembre de 1999 cuando la reducción fue de $200 \mathrm{pb}^{5}$. Notemos que durante el período de estudio se presentaron 18 movimientos en la tasa de subastas, 9 hacia abajo y 9 hacia arriba.

Finalmente, la Figura 3 contiene la evolución de la estructura a plazo de tasas de interés spot en Colombia estimadas por el método de Nelson y Siegel $(1987)^{6}$. Como se observa, los retornos que la integran muestran una caída de las tasas de interés hasta el final de 2005 con un repunte importante a lo largo del primer semestre de 2006, luego una caída cercana a los 200 pb-sobre todo en los

\footnotetext{
5 Para intervenir en el mercado de dinero, el Banco de la República tiene disponibles las tasas de subastas de expansión y contracción y lombardas de expansión y contracción.

${ }^{6}$ Para una primera aplicación del método de Nelson y Siegel al caso de Colombia véase Arango, Melo y Vásquez (2003). Se intentó trabajar con la estimación de la curva de cupón cero por el método de splines cúbicos pero las tasas resultaban, en extremo, volátiles.
} 
FIGURA 1

TASA DE INTERES DE POLITICA DEL BANCO DE LA REPUBLICA

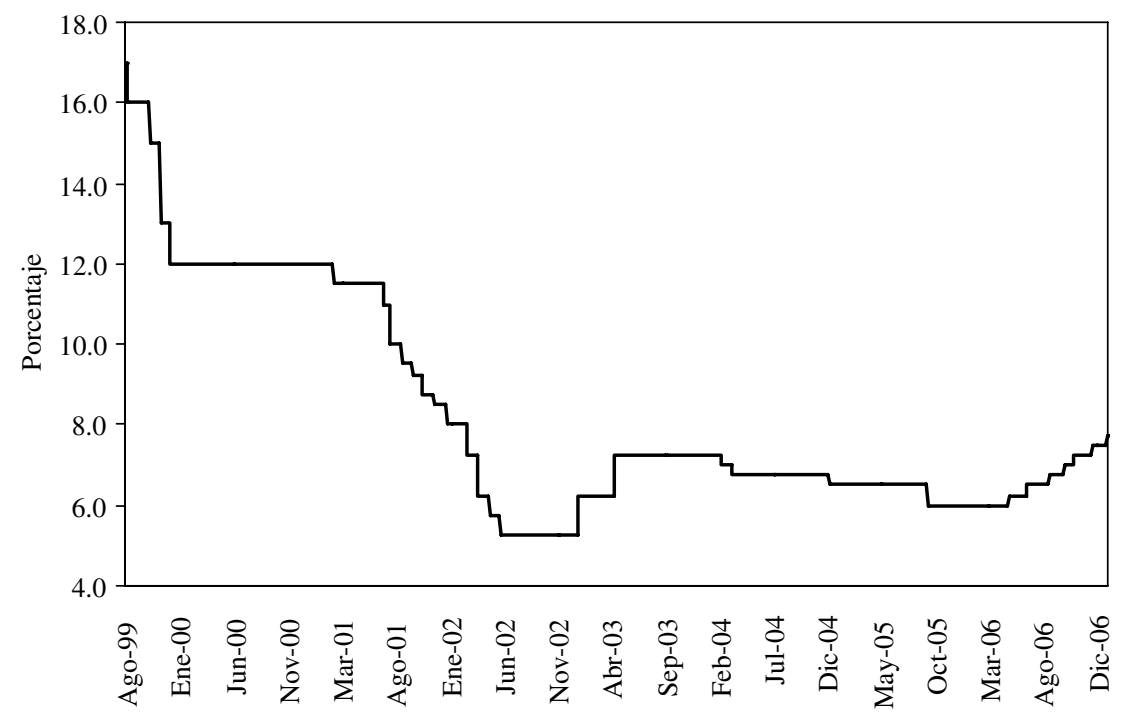

Nota: corresponde a la tasa de subasta (holandesa) de expansión.

FIGURA 2

MAGNITUD DE LA INTERVENCION DEL BR VISTA A TRAVES DE LA TASA DE SUBASTA DE EXPANSION

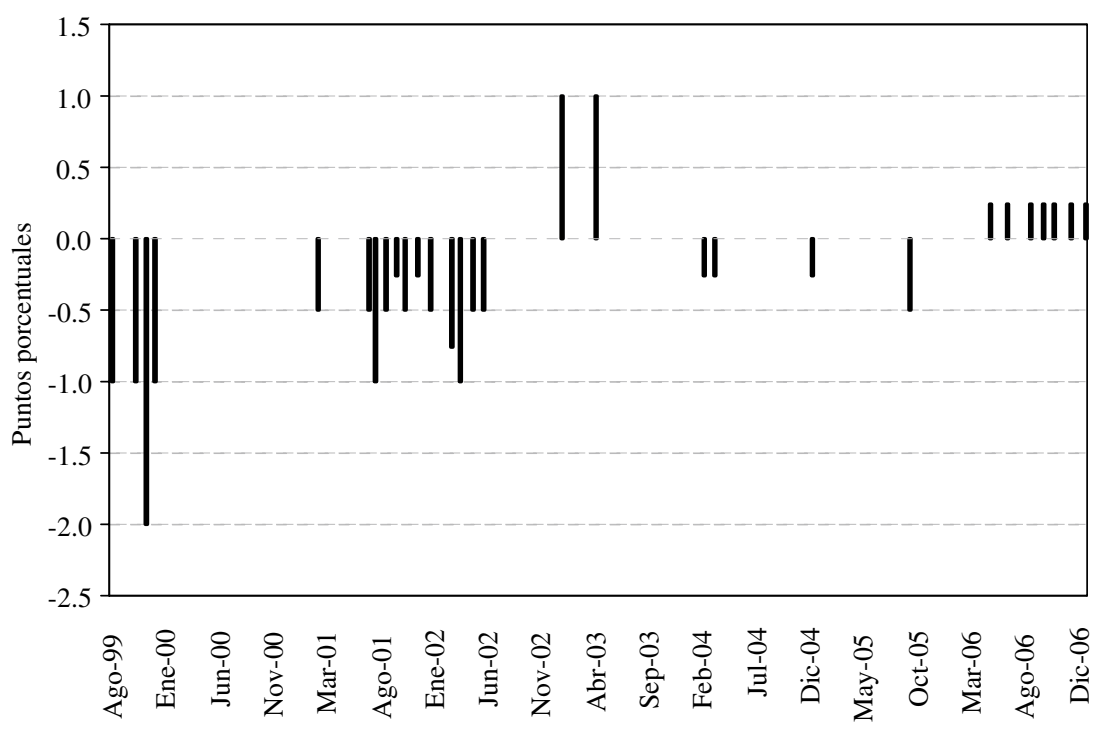


FIGURA 3

ESTRUCTURA A PLAZO DE LAS TASAS DE INTERES EN COLOMBIA Y MOVIMIENTOS DE LA TASA DE POLITICA

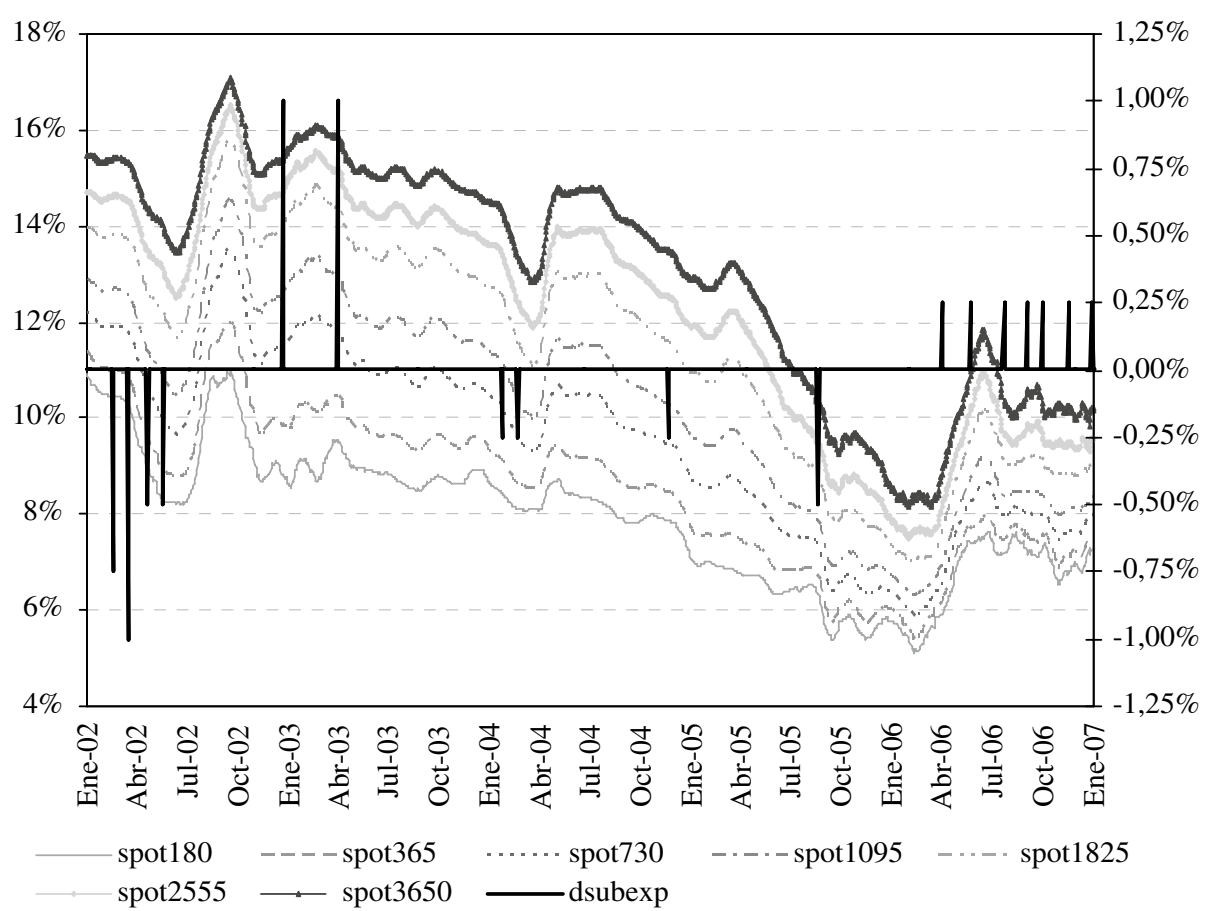

Nota: la figura presenta el promedio móvil 20 de las tasas spot, a distintos plazos (de 180 a 3650 días), de la curva cero cupón obtenida estimada mediante el método de Nelson y Siegel (1987). Corresponden a los títulos transados a través del Sistema Electrónico de Negociación (Banco de la República) y del Mercado Electrónico Nacional de la Bolsa de Valores de Colombia. La variable dsubexp representa el piso de la tasa de subasta (holandesa) de expansión para operaciones a un día.

títulos de mayor período al vencimiento- y, posteriormente, una relativa estabilización entre septiembre de 2006 y enero de 2007, pese a los cinco incrementos en las tasas de política que se registraron en dicho período ${ }^{7}$.

Se destaca el hecho de que la estructura a plazo tiene un comportamiento que no parece estar ligado -al menos exclusivamente-al de las tasas de política del BR. El movimiento diario de la estructura a plazo (Figura 3) sugiere la necesidad de controlar por otras variables. Este argumento lo explotamos más adelante al ampliar el enfoque de Cook y Hahn (1989) para tomar en cuenta el carácter de economía pequeña y abierta de Colombia.

${ }^{7}$ Puede decirse que hasta junio de 2006 la evolución de las tasas de interés de mercado venía coincidiendo con la de la tasa de subastas del BR y con el aumento en la liquidez de la economía señalado por algunos analistas (Escobar y Posada, 2004). 


\section{Movimientos en la Estructura a Plazo, Transparencia, Credibilidad e Hipótesis de EXPECTATIVAS}

En esta sección se presenta un vínculo entre la hipótesis de expectativas y los movimientos en la tasa de política. Para ello se utilizan la ecuación de Fisher y la hipótesis de expectativas. La primera plantea que la tasa de interés nominal, $i$, es, aproximadamente, igual a la tasa de interés real, $r$, más la inflación esperada, $\pi^{\mathrm{e}}$. Esto es:

$$
i_{t} \approx r_{t}+\pi_{t+1}^{e}
$$

La hipótesis de expectativas sugiere que la tasa de interés de los títulos a largo plazo, $i^{k}$, es igual a la media de las tasas forward de corto plazo, $f^{1}$ (como proxies de las tasas de interés esperadas). Esto es:

$$
i_{t}^{k}=\left[i_{t}^{1}+f_{t+1}^{1}+\ldots+f_{t+k-1}^{1}\right] / k
$$

Los dos postulados que ilustran las ecuaciones (1) y (2) permiten señalar que -bajo perfecta credibilidad de la autoridad monetaria- una política monetaria restrictiva (es decir, un aumento en las tasas de política) elevará, de manera transitoria, las tasas de interés de corto plazo (se encarece el dinero en todas las instancias del mercado) y reducirá las tasas de inflación de largo plazo. Este esquema es relativamente simple, ya que se espera que las señales que emite el BR al mercado de dinero al subir la tasa a la cual proporciona liquidez a un día a los intermediarios financieros se reflejen en las operaciones de crédito que se realizan entre éstos y el público. En ausencia de una prima de riesgo inflacionario (véase Arango y Flórez, 2008), el resultado será una disminución en las tasas de interés de los títulos a largo plazo, en el entendido que la autoridad monetaria no tiene la capacidad de alterar la tasa de interés real de largo plazo.

Sin embargo, la condición para obtener dichas reacciones es que haya alta credibilidad de la política monetaria ${ }^{8}$. Un banco central es creíble cuando los agentes creen que él hará lo que dice que va a hacer (Blinder, 2000) o, en términos de Cukierman (1986), un banco central es creíble si su política de perseguir estabilidad de precios, o más exactamente alcanzar su meta de inflación, es tomada por los agentes privados como la base para formar sus expectativas. Aunque existen diversos enfoques para abordar este aspecto ${ }^{9}$ creemos que argumentos como los

\footnotetext{
${ }^{8}$ El argumento de credibilidad se asocia a la discusión de "inconsistencia temporal de la política monetaria” y "reglas versus discreción” (Kydland y Prescott, 1977; Barro y Gordon, 1983).

${ }^{9}$ Véanse, por ejemplo, Ball (1995), Erceg y Levin (2003) o Céspedes y Soto (2005). Estos últimos, al estudiar el caso de una economía emergente como la chilena, introducen el tema de credibilidad por la vía de la información imperfecta sobre la meta de inflación de manera que los agentes, aunque están dotados de expectativas racionales, enfrentan un problema de extracción de señales al tratar de predecir la trayectoria futura de la tasa de inflación.
} 
de Haldane y Read (1999) son los adecuados para el presente trabajo; esto es, si la autoridad monetaria es creíble, las expectativas de inflación deberán moverse hacia la meta de largo plazo y en el mismo sentido deberán cambiar las tasas de interés nominal definidas para títulos cuyo plazo sea consistente con el horizonte de la meta.

A su vez, la credibilidad es un activo que se acumula por medio de mayor transparencia ${ }^{10}$. Algunos estudios empíricos han investigado el efecto de la mayor transparencia que requiere el esquema de metas de inflación. Sus hallazgos sugieren que la mayor transparencia genera una menor respuesta de los participantes del mercado a las medidas de política monetaria y una menor volatilidad de las tasas de interés de corto plazo, lo cual indica una mejor habilidad para predecir las acciones del banco central (Lyziak, Mackiewicz y Stanislawska, 2007; Haldane y Read, 2000).

Si los agentes están en posibilidad de predecir el comportamiento de la autoridad monetaria, lo anterior sugiere una respuesta como la que aparece en el Diagrama 1 donde $\Delta i_{t+k}$ y $\Delta \tilde{i}_{t, n}$ son, respectivamente, las variaciones a un día $\left(\Delta i_{t+k}=i_{t+k}-i_{t+k,-1}\right)$ en la tasa de retorno de títulos (cero cupón) que tienen vencimiento en $t+k$ períodos adelante y las variaciones de la tasa de política del banco central anticipadas $n$ días $\left(0<n<n^{\prime}\right.$, siendo $n^{\prime}$ la fecha de la anterior intervención); por lo tanto: $\Delta \tilde{i}_{t, n}=\tilde{i}_{t, n}-\tilde{i}_{t, n-1}$. En este sentido, se espera que la respuesta anticipada ( $n$ días) de las tasas de interés de mercado sea menor, o incluso nula, cuanto mayor sea el período de maduración de los títulos.

Otra forma conveniente de representar la reacción sobre la estructura a plazo de tasas de interés ante cambios en las tasas de política de la autoridad monetaria es utilizando el concepto de spread el cual identifica la diferencia en el retorno de títulos que vencen en dos horizontes diferentes de tiempo: $t+k \mathrm{y} t+j$ $(k>j)$; esto es: $i_{t, t+k}-i_{t, t+j}$. El Diagrama 2 muestra la reacción del spread ante un cambio en la tasa de política para diferentes combinaciones de vencimientos de los títulos $[(t+k)-(\underset{\sim}{t}+j)]$. De acuerdo con lo que acabamos de señalar, la curva $-\Delta\left[i_{t, t+k}-i_{t, t+j}\right] / \tilde{i}_{t, n}$ debe tener pendiente positiva.

\footnotetext{
10 Transparencia es un concepto multidimensional que incorpora aspectos políticos (apertura sobre los objetivos de política), económicos (información económica utilizada para la política monetaria: datos, modelos, pronósticos), de procedimiento (la forma en que son adoptadas las medidas de política: reglas, estrategias, minutas, votaciones), de política (oportunidad de los anuncios sobre las medidas), operacionales (implementación de las acciones de política: intervenciones de mercado) (véanse Eijffinger y Geraats, 2006; Geraats, 2002). Blejer, Ize, Leone y Werlang (2000) consideran la transparencia como un elemento clave del esquema de metas de inflación. El número 23 de European Journal of Political Economy editado por de Haan, Eijffinger y Rybiński, está dedicado a los temas de transparencia y credibilidad.
} 
DIAGRAMA 1

VARIACION ANTICIPADA EN LAS TASAS DE MERCADO ANTE UN CAMBIO EN LA TASA DE POLITICA

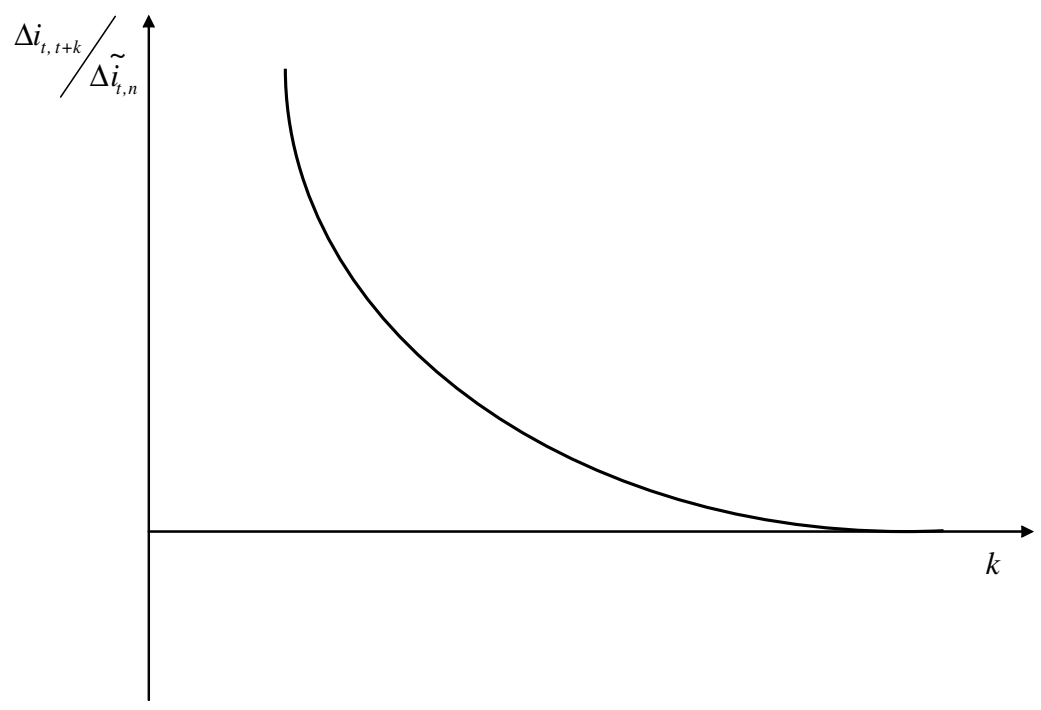

DIAGRAMA 2

RELACION ENTRE EL CAMBIO ANTICIPADO EN EL SPREAD Y LOS CAMBIOS EN LA TASA DE POLITICA

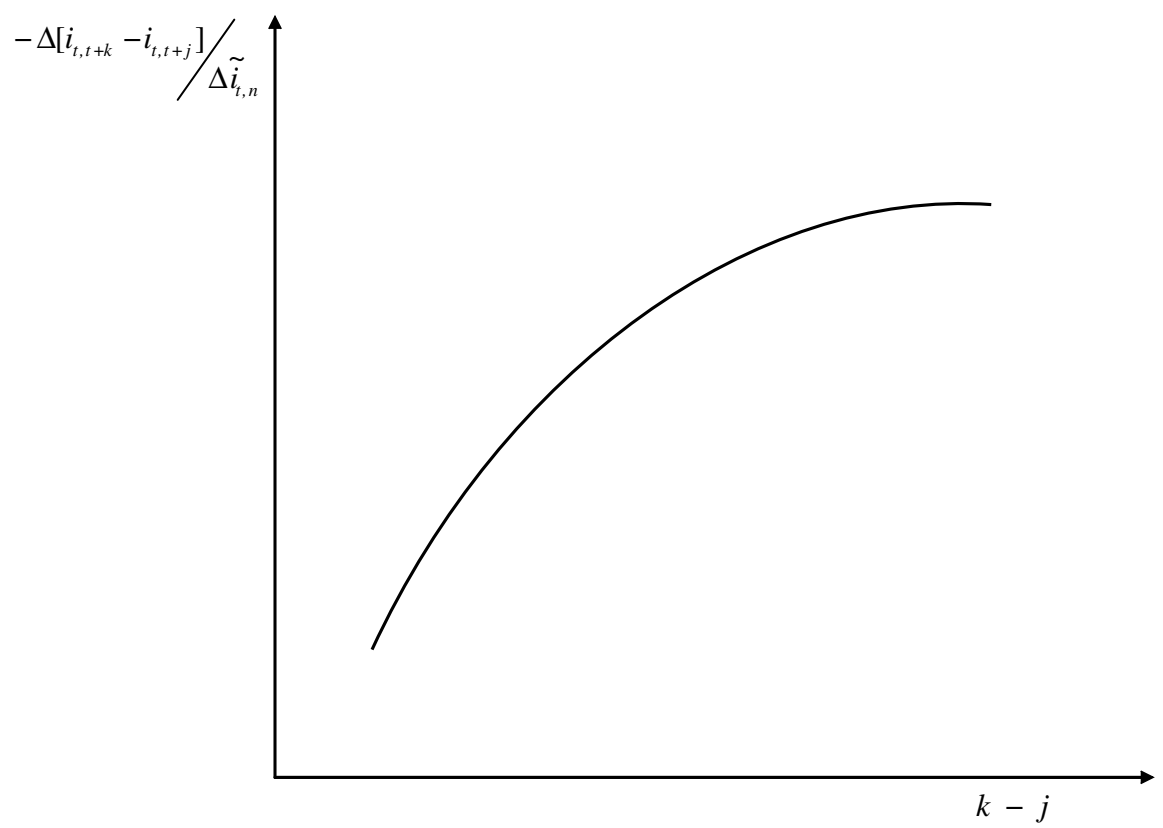




\section{Contribuciones y Enfoques Previos}

Cook y Hahn (1989, $\mathrm{CH}$ en lo que sigue) es la referencia fundamental en cuanto al efecto de las intervenciones de la autoridad monetaria -mediante movimientos de la tasa de interés de política- sobre la estructura a plazo. Dichos autores utilizaron los 76 cambios de la federal funds rate target (tasa de fondeo) publicados en el Wall Street Journal entre septiembre de 1974 y septiembre de 1979 para verificar la hipótesis de que el target tenía influencia sobre las tasas de interés de mercado. En particular, estos autores estimaron la respuesta de las tasas de los bonos a $\underset{\sim}{3}, 6$ y 12 meses y 3, 5, 7, 10 y 20 años a partir de la regresión $\Delta i_{t+k}=\alpha+\beta \Delta \tilde{i}_{t}+\mu_{t}$ donde $\Delta i_{t+k}$ y $\Delta \tilde{i}_{t}$ son las variaciones en la tasa de retorno de títulos que tienen vencimiento en el período $t+k$ y la tasa de política del banco central, respectivamente. Los hallazgos de $\mathrm{CH}$ sugieren que $\hat{\beta}>0$, con $\hat{\beta}$ decreciendo en la medida en que aumenta $k$.

La hipótesis anterior también fue verificada mediante la regresión $\Delta i_{t+k}=\alpha+\beta \Delta \tilde{i}_{t+n}+\mu_{t}$ para los títulos de tesorería a 3, 6 y 12 meses, siendo $n$ un número entero que va entre dos días antes y dos días después del cambio en las tasas de política $(-2 \leq n \leq 2)$. Los resultados no evidenciaron, salvo para el plazo de $k=12$ meses, dos días antes del cambio, ningún movimiento sistemático en las tasas de los papeles ni para uno ni para dos días antes de los cambios en el target. Sin embargo, el coeficiente de la tasa de fondeo un día después del cambio resultó significativo en los tres plazos considerados, mientras que para los dos días siguientes los cambios en la tasa resultaron significativos solamente para el plazo a 6 meses.

Se concluye, entonces, que las acciones de la Reserva Federal no pudieron ser anticipadas por los agentes (lo cual es síntoma de falta de transparencia) y que solamente estos pudieron reaccionar a los movimientos contemporáneos y anteriores en la tasa de fondeo. Cuando la Reserva Federal realizó operaciones de mercado abierto contraccionistas, las tasas de interés aumentaron en todos los plazos. Este resultado, interpretado a la luz del Diagrama 1, sugiere que si bien el parámetro $\hat{\beta}$ decrece en la medida en que el vencimiento de los títulos $(k)$ tiende a 20 años, no tiende a cero en la medida en que $k$ aumenta.

La interpretación convencional de los resultados de $\mathrm{CH}$, como también lo hacen Roley y Sellon (1995), es que los movimientos en $i_{t+k}$ se deben a cambios en las expectativas de que se modifique la tasa de captación a lo largo de la vida del título $^{11}$. Esta interpretación respalda la hipótesis de expectativas de la estructura a plazo de las tasas de interés (véase también Cook y Hahn, 1988).

\footnotetext{
${ }^{11}$ Romer y Romer (2000) sugieren, para explicar el resultado de $\mathrm{CH}$, que cuando la Reserva Federal restringe la liquidez los agentes interpretan que ésta tiene información desfavorable sobre el comportamiento de la inflación y, por lo tanto, revisan sus expectativas de inflación hacia arriba. Según los autores, el conjunto de información de la Reserva Federal es diferente del de los agentes (comercial forecasters) por lo que plantean un problema de información asimétrica. Para que su hipótesis tenga poder explicativo sobre los movimientos de las tasas de interés de largo plazo, es necesario suponer, además, que la Reserva Federal posee información útil sobre la inflación futura (sus pronósticos), que las acciones de política proveen señales de esta información y que los agentes responden a estas señales.
} 
En Inglaterra, Dale (1993) analizó la respuesta de las tasas de interés de mercado a los cambios de las tasas de interés oficiales del Banco de Inglaterra. Estudió el efecto de las treinta intervenciones en la Band 1 stop rate ${ }^{12}$ ocurridas entre comienzos de 1987 y julio de $1991^{13}$ en las tasas de títulos a siete vencimientos diferentes: 1, 3, 6 y 12 meses y 5, 10 y 20 años. La evidencia sugiere respuestas significativas en vencimientos que van de 1 mes a 5 años, quedando por fuera de dicha evidencia las tasas de títulos con vencimiento a 10 y 20 años.

Haldane y Read $(1999,2000)$ privilegian igualmente la hipótesis de expectativas para explicar los movimientos en la estructura a plazo ante cambios en las tasas de política. Plantean que las tasas de interés a plazo están determinadas por las expectativas de la trayectoria futura de las tasas de interés spot de corto plazo, las cuales son en buena medida determinadas por el banco central. Por lo anterior, la estructura a plazo depende de las creencias de los agentes acerca de la trayectoria esperada de las tasas del banco central actuales y futuras.

Estos autores sugieren la existencia de dos componentes de la regla de política monetaria sobre los cuales el sector privado podría tener información diferente de la que tienen las autoridades monetarias: la evolución de las variables macroeconómicas y los objetivos de política (también llamada información privada sobre las preferencias de las autoridades). De acuerdo con su interpretación, en casos de alta credibilidad y completa transparencia ${ }^{14}$, no debería registrarse ningún cambio en la estructura a plazo después de una modificación en las tasas oficiales. Agregan que una mayor transparencia atenúa la volatilidad de la curva en la parte corta, mientras que la credibilidad lo hace en la parte larga de la misma. Los autores presentan evidencia en el sentido de que bajo el régimen de inflación objetivo en el Reino Unido se ha reducido la magnitud de las respuestas de la estructura a plazo ante cambios en las tasas de política.

Romer y Romer (2000) utilizan un enfoque diferente que se apoya en la información de pronósticos tanto de la Reserva Federal como de los commercial forecasters. Su hipótesis es que las tasas de largo plazo reaccionan dado el superior conjunto de información de la autoridad monetaria sobre la trayectoria futura de inflación. De esta manera, cuando los agentes observan acciones asociadas a una política monetaria más restrictiva, en lugar de inferir que la FED está decidida a luchar contra la inflación, lo que intuyen es que ahora hay nueva información desfavorable sobre la inflación con la que antes no contaban. Sus resultados muestran que los pronósticos de la FED sobre la inflación son superiores a los de los comercial forecasters lo cual sugiere que aquella posee información útil sobre la inflación.

\footnotetext{
${ }^{12}$ Los tipos de interés Band 1 y Band 2 se referían a títulos con vencimientos inferiores a 14 días y a títulos con vencimientos entre 15 y 33 días, respectivamente.

${ }^{13}$ Debe notarse que el período muestral no cubre la adopción del esquema de metas de inflación en el Reino Unido la cual se produjo en octubre de 1992.

${ }^{14}$ Sobre estos aspectos véanse el texto de Blejer, Ize, Leone y Werlang (2000). De Haan, Eijffinger y Rybinsky (2007) y los demás artículos de ese número están dedicados al tema de la transparencia y la comunicación de los bancos centrales.
} 
Kuttner (2001) provee resultados en los que se enfatiza la respuesta de la estructura a plazo a movimientos anticipados y no anticipados de la federal fund rate target. Según Kuttner, la respuesta de las tasas de interés de mercado a cambios esperados es pequeña mientras que la respuesta a cambios no anticipados es mucho mayor. Finalmente, Demiralp y Jorda (2004), siguiendo la línea de Kuttner, descomponen las intervenciones entre esperadas y sorpresivas y concluyen que desde 1994, año en el que se produjo un cambio en la manera de proveer información sobre las medidas de política, los anuncios del Federal Open Market Commitee (FOMC) permiten al mercado anticipar mejor la naturaleza y el momento de las intervenciones futuras ${ }^{15}$.

Los estudios anteriores se concentran en observar si los movimientos en las tasas de política afectan la estructura a plazo tomando en cuenta, en algunos casos, las acciones previstas y no previstas de política. Sin embargo, dejan de lado otros determinantes que es en lo que este documento se interesa en controlar.

\subsection{Resultados iniciales bajo el enfoque de Cook y Hahn}

En primera instancia se estima la expresión planteada por $\mathrm{CH}$, la cual se re-escribe como:

$$
\Delta i_{t, t+k}=\alpha_{k 0}+\alpha_{k 1} \Delta \tilde{i}_{t}+\mu_{k t}
$$

donde $\Delta i_{t, t+k}=i_{t, t+k}-i_{t, t+k,-1}$ son los cambios diarios en la tasa spot efectiva anual derivada de la curva cero cupón del mercado secundario de deuda pública denominada en pesos con vencimiento en $t+k, \Delta \tilde{i}_{t}$ son los cambios en la tasa de política del BR y $\mu_{k t}$ es una perturbación de media cero y varianza constante.

Este trabajo se concentra en los vencimientos ocurridos en $(t+k)$, los cuales están dados en días (años), así: 3.650 (10 años), 2.555 (7 años), 1.825 (5 años), 730 ( $1 \frac{1}{2}$ años), 365 ( 1 año) y 180 ( $\frac{1}{2}$ año). Los resultados de las regresiones se muestran en el Cuadro 1.

15 Mehra (1994) propone un enfoque de cointegración y corrección de errores para encontrar los determinantes de las tasas de interés de largo plazo. 
CUADRO 1

ESTIMACION DE LA EXPRESION: $\Delta i_{t, t+k}=\alpha_{k 0}+\alpha_{k 1} \Delta \tilde{i}_{t}+\mu_{k t}$

\begin{tabular}{ccc|cc}
\hline & \multicolumn{2}{c|}{ Tasas spot } & \multicolumn{2}{c}{ Tasas forward } \\
\cline { 2 - 5 } Vencimiento & $\hat{\alpha}_{k 1}$ & $H_{0}: \alpha_{k 1}=0$ & $\hat{\alpha}_{k 1}$ & $H_{0}: \alpha_{k 1}=0$ \\
\hline \multirow{2}{*}{3.650} & 0,2727 & 0,224 & 0,4073 & 0,253 \\
2.555 & 0,2167 & 0,224 & 0,8770 & 0,256 \\
1.825 & 1,6004 & 0,345 & 0,3167 & 0,276 \\
730 & 0,0797 & 0,583 & 0,0992 & 0,612 \\
365 & 0,0864 & 0,579 & 0,0584 & 0,714 \\
180 & 0,1084 & 0,515 & 0,0786 & 0,617 \\
\hline
\end{tabular}

Según se desprende del Cuadro, los movimientos de la tasa de política no tienen, de acuerdo con la significancia estadística, efectos contemporáneos ni en las tasas spot ni en la forward ${ }^{16}$. El ejercicio también se realizó con el cambio en la tasa spot desde cinco días antes hasta cinco días después del movimiento de la tasa de política del BR. En este caso, la expresión (3) cambia ligeramente para convertirse en: $\Delta i_{t, t+k}=\alpha_{k 0}+\alpha_{k 1} \Delta i_{t \pm n}+\mu_{k t}$ donde $n=1,2,3,4$, y 5. Los resultados aparecen en el Cuadro 2 tanto para las tasas spot como para las forward. De acuerdo con los mismos, no hay -salvo por tres excepciones-ninguna reacción en las tasas de interés del mercado ante movimientos inminentes próximos (antes de cinco días) o pasados (después de cinco días) de la tasa de política del BR.

La falta de validez empírica del enfoque de $\mathrm{CH}$, para el caso de Colombia, se puede deber al tamaño muestral y al poco número de intervenciones del BR registradas durante ese período, pero también a una alta credibilidad del BR durante el período de análisis dado el esquema de metas de inflación o a que los cambios en la tasa de política son anticipados (descontados) antes de cinco días o tenidos en cuenta después de cinco días e incorporados en las tasas de mercado. También es posible vincular este resultado a que no se cumple la teoría de expectativas (en lo que tiene que ver con los tramos intermedio y largo de la curva) y que más bien efectos relativos las primas de liquidez pueden estar afectando los resultados. Finalmente, es posible que en el corto plazo no haya conexión entre las tasas de política y las tasas del mercado secundario de deuda pública. En otras palabras, bajo este enfoque empírico, aún no se están incorporando los determinantes fundamentales de los movimientos o cambios de pendiente de la estructura a plazo.

Nótese, en concordancia con lo anterior, que la estructura a plazo de Colombia se mueve permanentemente aun cuando la autoridad monetaria no esté modificando o no esté considerando modificar la tasa de política. Por tal razón, en la siguiente sección se sigue un enfoque que amplía el de $\mathrm{CH}$ para una economía

\footnotetext{
16 Dado el reducido número de observaciones, los resultados deben tomarse con el mayor cuidado. Los errores estándar se obtuvieron mediante un boostraping de 10.000 replicaciones.
} 


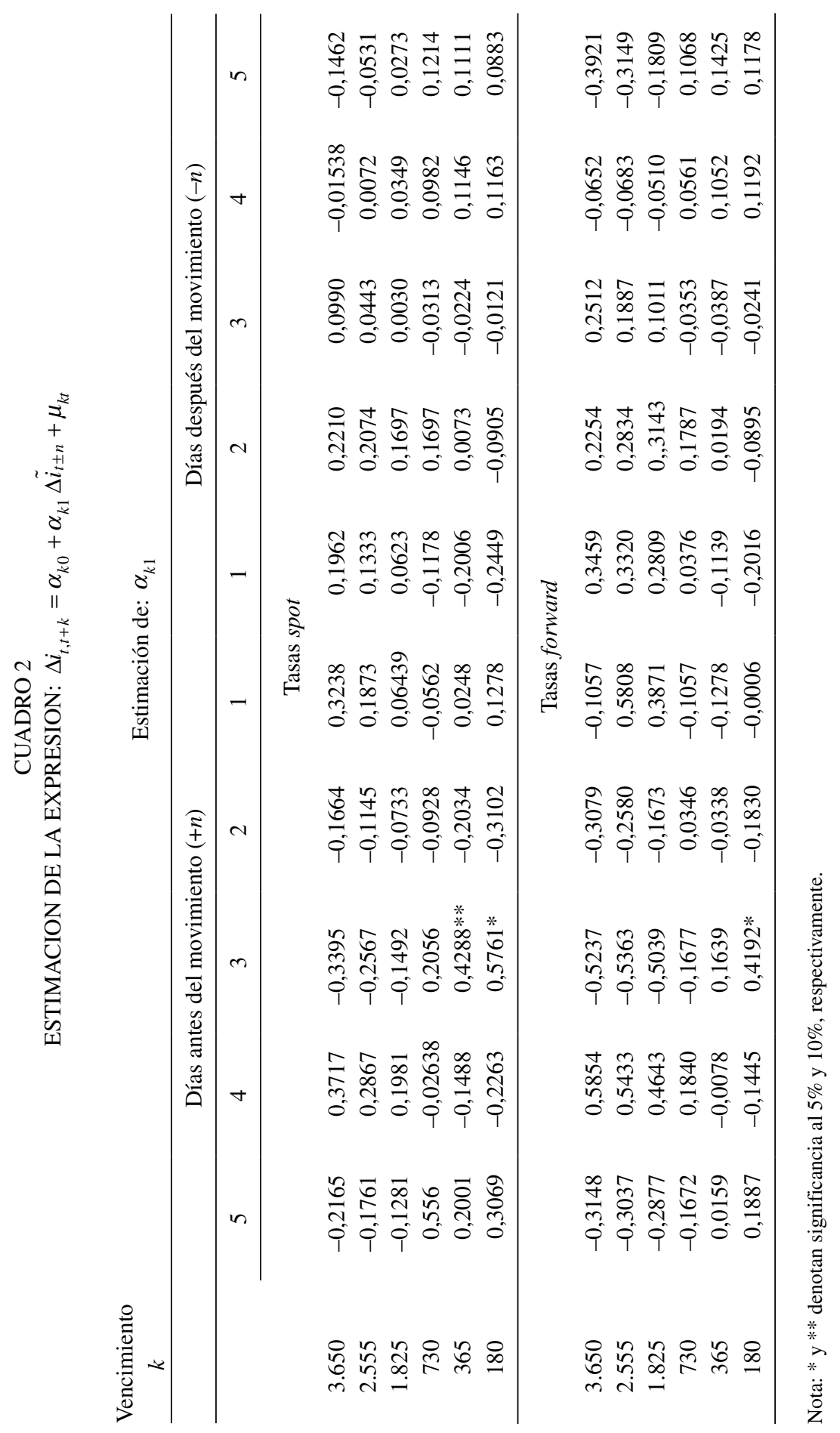


emergente que cuenta con un mercado que, aunque es poco profundo como el colombiano, está ligado a los mercados externos.

\section{EnFoQue TeóRICO}

El enfoque de $\mathrm{CH}$ invocado en la sección anterior tiene validez, posiblemente, para mercados profundos como los de Estados Unidos, el Reino Unido, Alemania, etc. Pero aun así, surge la pregunta: ¿por qué se mueven las curvas de rendimientos en los días en que no intervienen las autoridades monetarias?

Teniendo presente este interrogante, a continuación se plantea un modelo en el que, bajo el supuesto de movilidad de capitales, se somete a verificación el cumplimiento de la hipótesis de paridad descubierta de intereses ${ }^{17}$, con agentes que tienen aversión al riesgo. Esto es:

$$
\Delta S_{t, t+1}^{E}=\beta_{0}+\beta_{1}\left(i_{t, t+k}-i_{t, t+k}^{*}\right)-\beta_{2} \rho_{k, t}
$$

siendo $\Delta S_{t, t+1}^{E}$ la tasa de depreciación anual (entre $t$ y $t+1$ ) esperada, condicional al conjunto de información disponible al finalizar el período $t, \Omega_{t} ; i_{t, t+k}^{*}$ la tasa de interés externa para un título con maduración en $t+k,\left(i_{t, t+k}-i_{t, t+k}^{*}\right)$ el spread de tasas de interés construido con base en títulos que vencen $k$ períodos adelante, $\rho_{k, t}$ la prima de riesgo asociada a dichos títulos y $\beta_{0}, \beta_{1}$ y $\beta_{2}$ son parámetros cuyos valores esperados, bajo la hipótesis de paridad descubierta de intereses, son $\beta_{0}=0, \beta_{1}=1$ y $\beta_{2}=1$. La expresión (3) se puede reescribir como:

$$
i_{t, t+k}=\alpha_{k 0}+\alpha i_{t, t+k,-1}+\alpha_{k 1} \Delta \tilde{i}_{t}+\mu_{k t}
$$

donde se espera que $\alpha=1$. Retrasando (4) un día y resolviendo para $i_{t, t+k,-1}$ se obtiene:

$$
i_{t, t+k,-1}=-\frac{\beta_{0}}{\beta_{1}}+\frac{1}{\beta_{1}} \Delta S_{t, t+1,-1}^{E}+i_{t, t+k,-1}^{*}+\frac{\beta_{2}}{\beta_{1}} \rho_{k, t,-1}
$$

y reemplazando (6) en (5) se llega a:

$$
i_{t, t+k}=\alpha_{k 0}+\alpha\left[-\frac{\beta_{0}}{\beta_{1}}+\frac{1}{\beta_{1}} \Delta S_{t, t+1,-1}^{E}+i_{t, t+k,-1}^{*}+\frac{\beta_{2}}{\beta_{1}} \rho_{k, t,-1}\right]+\alpha_{k 1} \Delta \tilde{i}_{t}+\mu_{k t}
$$

que es una de las ecuaciones que estimaremos.

17 Para una explicación sencilla de esta hipótesis véase Isard (1996); sobre alternativas de verificación empírica véanse McCallum (1994) y, más recientemente, Boudoukh, Richardson y Whitelaw (2005). 
Haciendo, para los títulos que vencen $j$ períodos adelante, el mismo desarrollo que se hizo para los que vencían $k$ períodos adelante, y que permitió llegar a (7), se obtiene:

$$
i_{t, t+j}=\alpha_{j 0}+\alpha\left[-\frac{\beta_{0}}{\beta_{1}}+\frac{1}{\beta_{1}} \Delta S_{t, t+1,-1}^{E}+i_{t, t+j,-1}^{*}+\frac{\beta_{2}}{\beta_{1}} \rho_{j, t,-1}\right]+\alpha_{j 1} \Delta \tilde{i}_{t}+\mu_{j t}
$$

y restando (8) de (7) se obtiene una expresión para el spread que permite verificar el efecto de las acciones de política del BR controlando por la paridad descubierta de intereses. Esto es:

$$
\begin{aligned}
i_{t, t+k}-i_{t, t+j}= & \left(\alpha_{k 0}-\alpha_{j 0}\right)+\alpha\left[i_{t, t+k,-1}^{*}-i_{t, t+j,-1}^{*}\right]+\alpha \frac{\beta_{2}}{\beta_{1}}\left[\rho_{k, t,-1}-\rho_{j, t,-1}\right. \\
& +\left[\alpha_{k 1}-\alpha_{i 1}\right] \Delta \hat{i}_{t}+\left[\mu_{k t}-\mu_{i t}\right]
\end{aligned}
$$

que es la otra expresión que se estima más adelante.

En este trabajo se consideran como plazos largos $(k)$, en días, los siguientes: $3.650 ; 2.555$ y 1.825 y como plazos cortos $(j): 365$ y 180 . En la Figura 4 se observa el comportamiento de los diferenciales de tasas de interés internos y externos, destacándose la alta volatilidad de las tasas internas frente a las tasas correspondientes de Estados Unidos.

Para estimar la ecuación (5) se requiere la serie de expectativas de devaluación del día anterior, $\Delta S_{t, t+1,-1}^{E}$. Para estimar esa variable -no observable en frecuencia diaria para el período muestral de este trabajo- se utilizarán tres alternativas diferentes: en primer lugar, se utiliza la serie de tasa de devaluación esperada que se deriva del mercado a plazo del dólar (en la Figura 5 se denomina "Mercado forward") ${ }^{18}$. En segundo lugar, se genera una serie suponiendo que los agentes esperan que la devaluación entre $t$ y $t+1$ sea igual a la devaluación que se presentó entre $t-1$ y $t$; este mecanismo se designa devaluación vencida (DEV_VENCIDA/ MES). Finalmente, se utiliza un mecanismo de devaluación que se construye como un promedio móvil centrado de 241 días de la tasa de devaluación vencida que se identifica como mecanismo de promedio móvil (DEV_MA/MES).

La Figura 5, además de estos mecanismos de expectativas de devaluación, incluye también las expectativas que se derivan de la encuesta que realiza en BR mensualmente -desde el año 2003- a un grupo de instituciones nacionales (sobre la devaluación esperada); esta variable se denomina Encuesta. Finalmente, la Figura 5 también incluye la devaluación observada un año adelante ${ }^{19}$.

\footnotetext{
18 Esta alternativa nos lleva de la paridad descubierta de intereses a la paridad cubierta.

${ }^{19}$ Un punto importante acá es que la serie se genera originalmente en frecuencia diaria, pero para realizar la comparación y construir las series que aparecen en la Figura 5 se calcula el promedio mensual de Mercado_forward, DEV_VENCIDA y DEV_MA.
} 


\section{FIGURA 4 \\ SPREAD DE TASAS DE INTERES DOMESTICAS Y EXTERNAS}

Títulos de endeudamiento doméstico

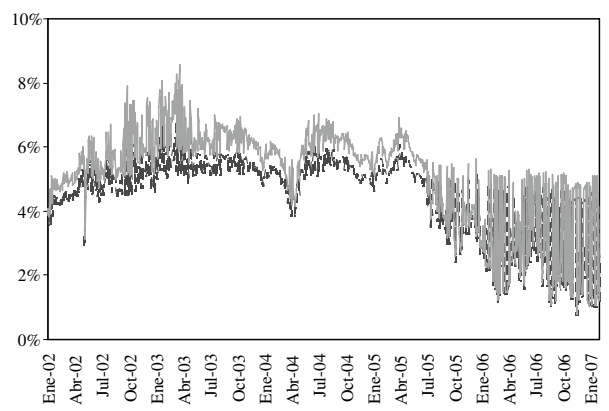

----TES3650_365

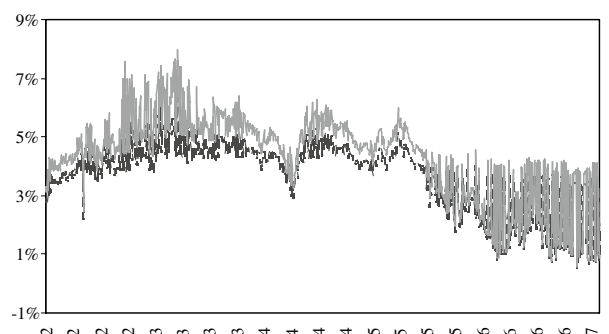

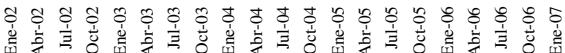
----TES2555_365

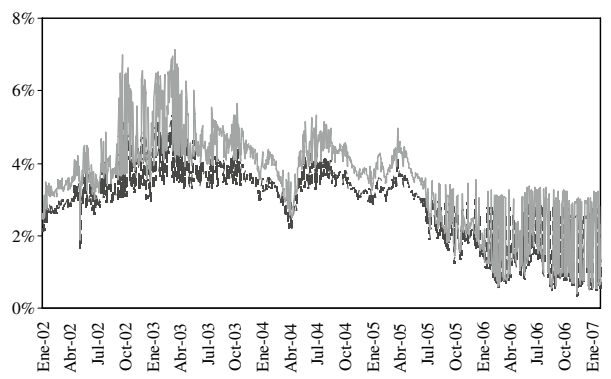

----TES1825_365
TES2555_180

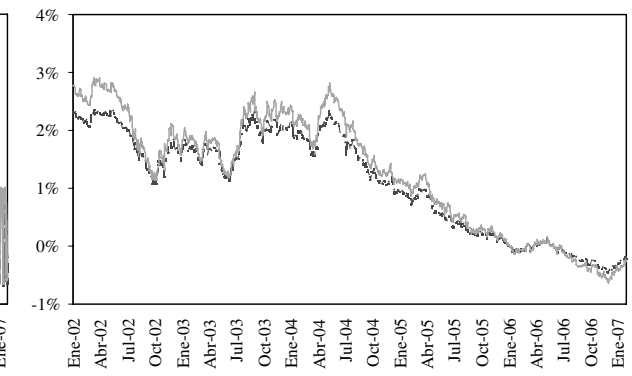

- - - - Treasuries2555_365—Treasuries2555_180
Títulos de endeudamiento externo

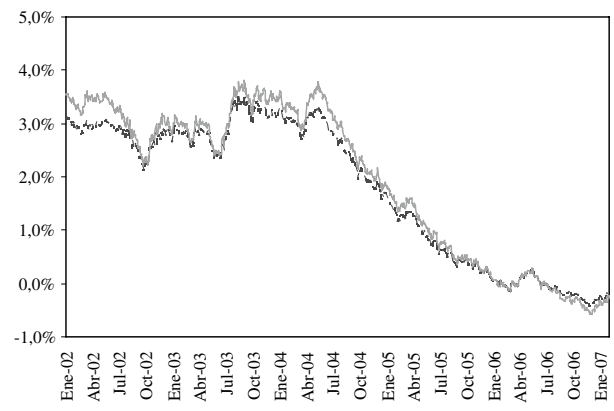

-.-- Treasuries3650_365

— Treasuries3650_180

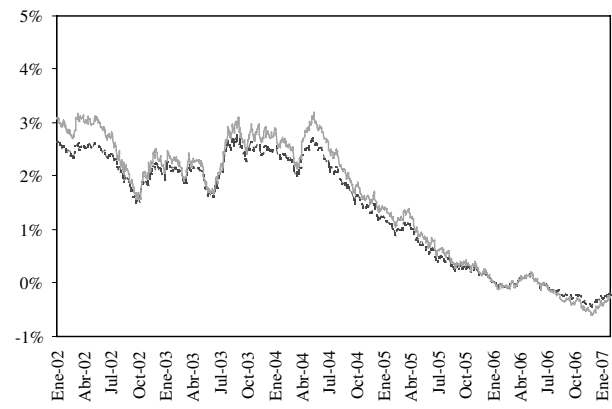

----Treasuries1825_365

- Treasuries1825_180

Fuente: Bloomberg (I025 index) y Banco de la República. 
FIGURA 5

TASAS DE DEVALUACION ESPERADAS Y OBSERVADA

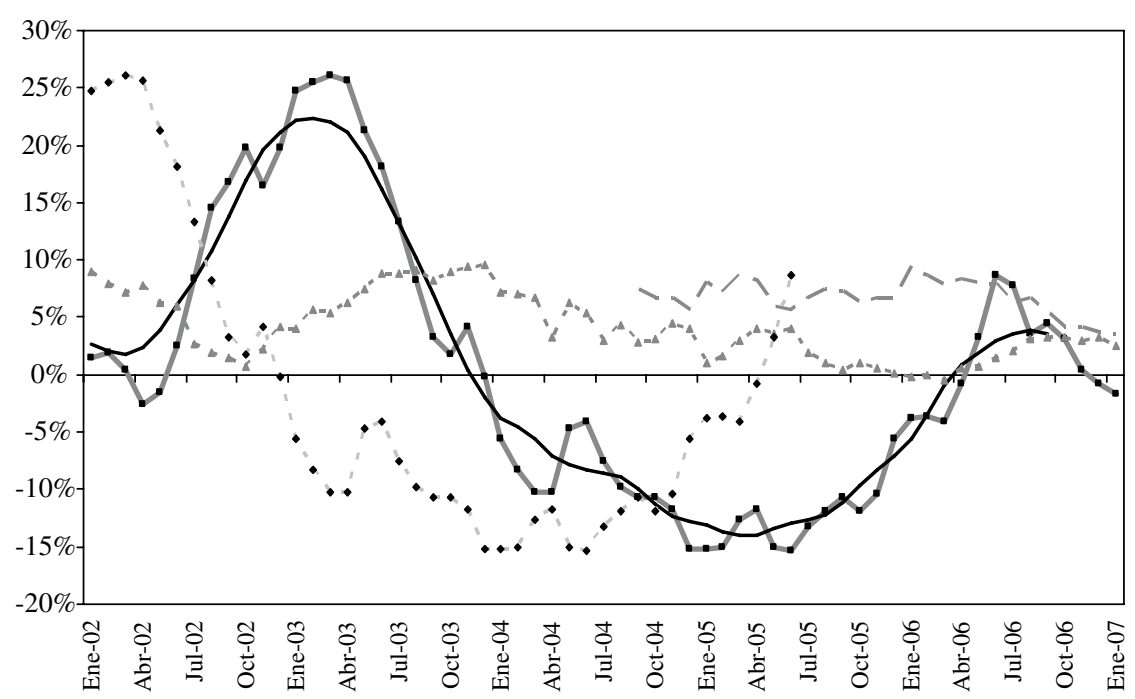

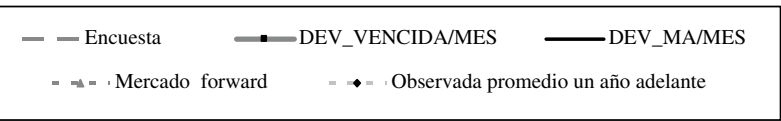

Nota: Encuesta son las expectativas de devaluación construidas a partir de la encuesta de expectativas del BR; DEV_VENCIDA/MES supone que la devaluación esperada entre $t$ y $t+1$ es igual a la que se presentó entre $t-1$ y $t$; DEV_MA/MES representa la devaluación esperada construida como el promedio móvil centrado de 241 días; mercado forward representa la serie de devaluación implícita en las negociaciones de divisas realizadas en el mercado forward. Todas las variables están situadas un año adelante.

De acuerdo con la Figura 5, desde el momento en que se tienen expectativas de devaluación un año adelante de la encuesta del BR (septiembre de 2004), se observa poca correspondencia entre las mismas y los mecanismos de devaluación vencida (DEV_VENCIDA/MES) y de media móvil (DEV_MA/MES). Más aún, las tres series se apartan tanto de las expectativas de devaluación derivadas del mercado forward como de la devaluación observada. Inclusive, estas dos últimas son muy diferentes entre sí.

\section{ENFOQUe EMPÍRICO y Resultados}

Las estimaciones de este trabajo se hicieron utilizando variables tanto en frecuencia diaria como semanal. A continuación se presentan los resultados obtenidos. 


\subsection{Resultados en frecuencia diaria}

En primera instancia deseamos estimar la ecuación (7) que ahora se renumera como (10) por el cambio que significa trabajar con $\rho_{t,-1}$, que para nuestro caso será el $\mathrm{EMBI}^{+}$de Colombia, en lugar de $\rho_{k, t,-1}$, ya que no se dispone de una prima de riesgo asociada a cada período de vencimiento de los títulos. Esto es:

$$
i_{t, t+k}=\alpha_{k 0}+\alpha\left[-\frac{\beta_{0}}{\beta_{1}}+\frac{1}{\beta_{1}} \Delta S_{t, t+1,-1}^{E}+i_{t, t+k,-1}^{*}+\frac{\beta_{2}}{\beta_{1}} \rho_{t,-1}\right]+\alpha_{k 1} \Delta \tilde{i}_{t}+\mu_{k t}
$$

Siguiendo las prácticas usuales, para verificar las hipótesis de interés, $H_{o}: \alpha_{k 1}=0, H_{o}: \alpha=1$ y $H_{o}: \alpha \beta_{2} / \beta_{1}=1$, se utilizan tanto las tasas de interés spot como las forward del mercado colombiano. Para efectos empíricos se utilizaron los datos de la curva cero cupón estimados, por el método de Nelson y Siegel (1987), para el caso de las tasas domésticas, y la serie I025 index de Bloomberg que identifica tasas spot, cero cupón, del mercado de Estados Unidos.

Dado que se obtuvo evidencia de que las series pertinentes de la ecuación (10), es decir, $i_{t, t+k}, i_{t, t+k,-1}^{*}$ y $\rho_{t,-1}$ son variables $I(1)$, se utilizó un enfoque de cointegración. En particular, la expresión (10) fue estimada mediante el método de estimación de mínimos cuadrados ordinarios completamente modificados (FMOLS) de Phillips y Hansen (1990) para sistemas cointegrados (véase Hamilton, 1994, página 613).

Los resultados obtenidos, que se complementan con la prueba de cointegración de Shin (1994), sugieren que no hay evidencia de cointegración bajo ninguno de los mecanismos de expectativas ni en frecuencia diaria ni en frecuencia semanal. Tampoco hay evidencia del cumplimiento de la hipótesis de paridad descubierta de intereses ${ }^{20}$. Por tal razón, se continúa con el contraste empírico modificando la especificación de la ecuación a estimar. Ahora el trabajo se concentra en la ecuación (9) donde el spread doméstico está explicado por el spread externo, el diferencial de riesgo entre los períodos $k$ y $j$ y las intervenciones del BR.

Dicha ecuación requiere de la construcción de primas de riesgo asociadas a cada período de vencimiento, $\rho_{k}$ donde $k=3.650,2.555,1.825, \mathrm{y} \rho_{j}$ donde $j=365$ y 180. Para efectuar esa construcción se utiliza la ecuación:

$$
\rho_{k, t,-1}=\beta_{0} / \beta_{2}+\beta_{1} / \beta_{2}\left[i_{t, t+k,-1}-i_{t, t+k,-1}^{*}\right]-1 / \beta_{2} \Delta S_{t, t+1,-1}^{E}
$$

la cual se obtiene de la ecuación (6) ${ }^{21}$. Las primas fueron estimadas imponiendo las restricciones teóricas de la hipótesis de paridad descubierta de intereses (i.e., $\beta_{0}=0, \beta_{1}=1$ y $\left.\beta_{2}=1\right)$.

\footnotetext{
${ }^{20}$ Cubierta en el caso del mercado forward.

${ }^{21}$ En este caso, la prima de riesgo resulta endogenizada, aunque predeterminada por estar rezagada.
} 
Observemos que la ecuación (11) utiliza las expectativas de devaluación, las cuales hemos aproximado por las tres vías mencionadas antes: el implícito en el mercado forward, la devaluación vencida y un promedio móvil. La Figura 6 presenta las primas de riesgo correspondientes al plazo de 3.650 días que resultan de utilizar cada uno de los tres mecanismos de expectativas. Con ellas se presenta también la prima de riesgo $\mathrm{EMBI}^{+}$de Colombia.

De nuevo, se obtuvo evidencia de que las series $i_{t, t+k}-i_{t, t+j},\left[i_{t, t+k,-1}^{*}-i_{t, t+j,-1}^{*}\right]$ y $\left[\rho_{k, t,-1}-\rho_{j, t,-1}\right]$ son $I(1)$. En concordancia con la estimación de la ecuación (7), se utilizan mínimos cuadrados ordinarios completamente modificados (FMOLS) de Phillips y Hansen (1990) para sistemas cointegrados.

\section{FIGURA 6 \\ PRIMAS POR RIESGO ESTIMADAS DADA UNA \\ TASA DE DEVALUACION ESPERADA}

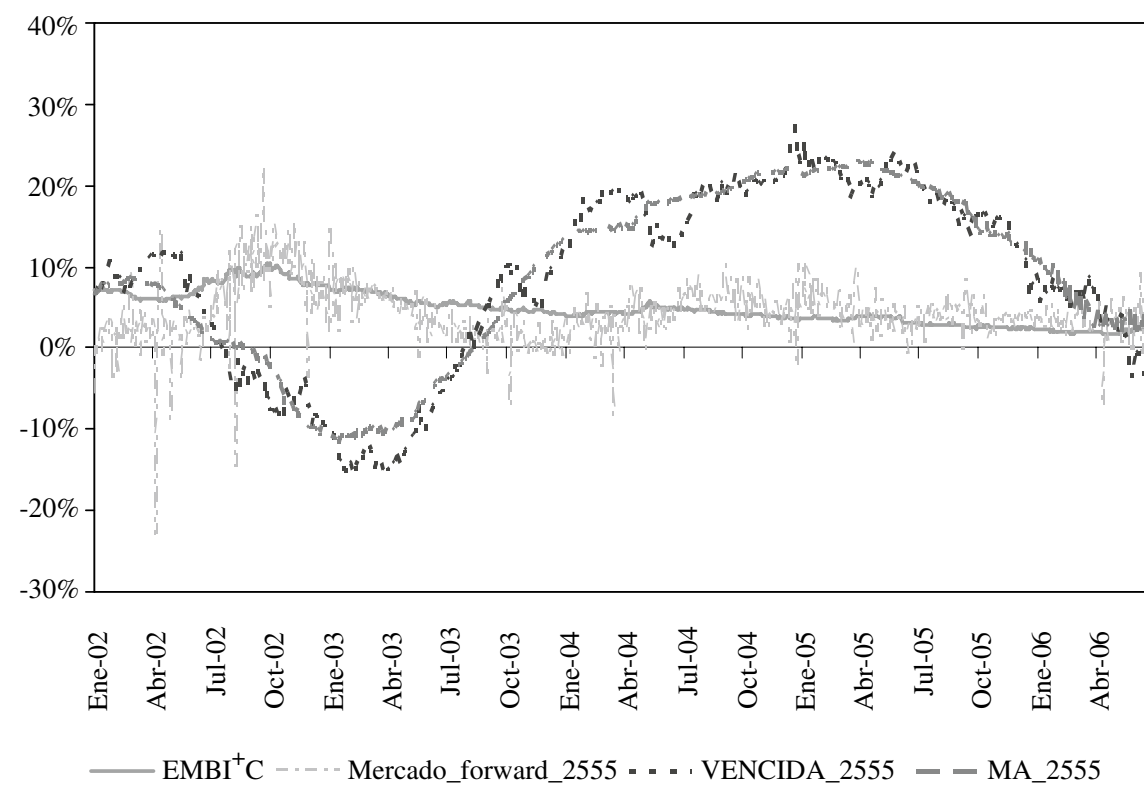

Nota: $\mathrm{EMBI}^{+} \mathrm{C}$ identifica la prima de riesgo que lleva dicho nombre; VENCIDA_2555 es la prima de riesgo implícita cuando las expectativas del mecanismo de devaluación es el vencido; MA_2555 representa la prima de riesgo implícita cuando las expectativas de devaluación se construyen como el promedio móvil centrado de 241 días; y, Mercado_forward_identifica la prima de riesgo estimada cuando las expectativas de devaluación son derivadas del mercado forward de divisas.

La Tabla 3 presenta los resultados de la estimación de la ecuación (9) en frecuencia diaria. Allí se destacan varios hechos. En primer lugar, la construcción de las primas de riesgo utilizando la ecuación (11) hace inocuo el mecanismo de expectativas de devaluación utilizado para estimar las primas de riesgo, ya que al 
tomar la diferencia de las ecuaciones para $k$ y $j$ las expectativas de devaluación desaparecen; esto es:

$$
\rho_{k, t,-1}-\rho_{j, t,-1}=\left[i_{t, t+k,-1}-i_{t, t+k,-1}^{*}\right]-\Delta S_{t, t+1,-1}^{E}-\left[i_{t, t+j,-1}-i_{t, t+j,-1}^{*}\right]+\Delta S_{t, t+1,-1}^{E}
$$

Por ello, es indiferente estimar la ecuación (9) con cualquiera de los tres mecanismos de expectativas de devaluación; en consecuencia el Cuadro 3 no distingue los mecanismos de expectativas vencidas del derivado del mercadoforward, ya que los resultados son idénticos. Para el caso de las primas calculadas con base en las expectativas de devaluación de media móvil, los resultados son diferentes simplemente porque el período muestral cambia; se dispone de 120 observaciones menos que en los otros dos mecanismos.

En segundo lugar, en relación con los spread de tasas de interés externas, los coeficientes estimados son positivos, significativos y cercanos a la unidad lo cual es un síntoma del cumplimiento de la hipótesis de paridad descubierta de intereses $^{22}$. Asimismo, los coeficientes del diferencial de primas de riesgo son positivos y significativos.

Finalmente, los coeficientes asociados a la variación en las tasas de política no son significativos ${ }^{23}$. Esto sugiere que los movimientos en la tasa de política del BR no logran generar reacción contemporánea alguna en las tasas de mercado. Sin embargo, llama la atención que la respuesta (aun cuando no significativa) es siempre superior para los spreads calculados con base en 365 días que para los calculados con base en 180 días; es decir, en la cuarta columna de coeficientes, $\left[\alpha_{3650}-\alpha_{180}\right]>\left[\alpha_{3650}-\alpha_{365}\right]$, ambos asociados, desde luego, a la variable $\Delta \tilde{i}_{t}$ lo cual es síntoma de que la reacción es mayor en las tasas de títulos a 365 que en las de títulos a 180 días. Este hallazgo va en contra de la hipótesis de expectativas de tasas de interés.

El Cuadro 4 presenta los resultados de la estimación del coeficiente asociado a los cambios en la tasa de política adelantado y rezagado cuatro días procurando capturar algún resultado sintomático de las expectativas de movimientos de la misma o de alguna reacción retardada en el mercado. Sin embargo, dichos coeficientes no resultaron significativos.

\footnotetext{
22 Por el álgebra y la manipulación realizada podría decirse que lo único que se está verificando es que las tasas de interés interna y externa tienen raíz unitaria. Sin embargo, aun para que esto fuera cierto se requeriría que $\beta_{2} / \beta_{1}=1$, lo cual, igualmente, es síntoma del cumplimiento de la hipótesis de paridad descubierta de intereses.

23 Podría estar sucediendo que la reacción de las tasas sea la misma en todos los plazos; es decir, que los retornos de los títulos a distintos vencimientos se muevan en forma horizontal (paralela) ante movimientos en la tasa de política del BR. Sin embargo, el análisis de los resultados de las estimaciones de la ecuación (7) permitiría descartar dicha hipótesis.
} 


\section{CUADRO 3 \\ ESTIMACION DE LA ECUACION (9) EN FRECUENCIA DIARIA CON TASAS SPOT}

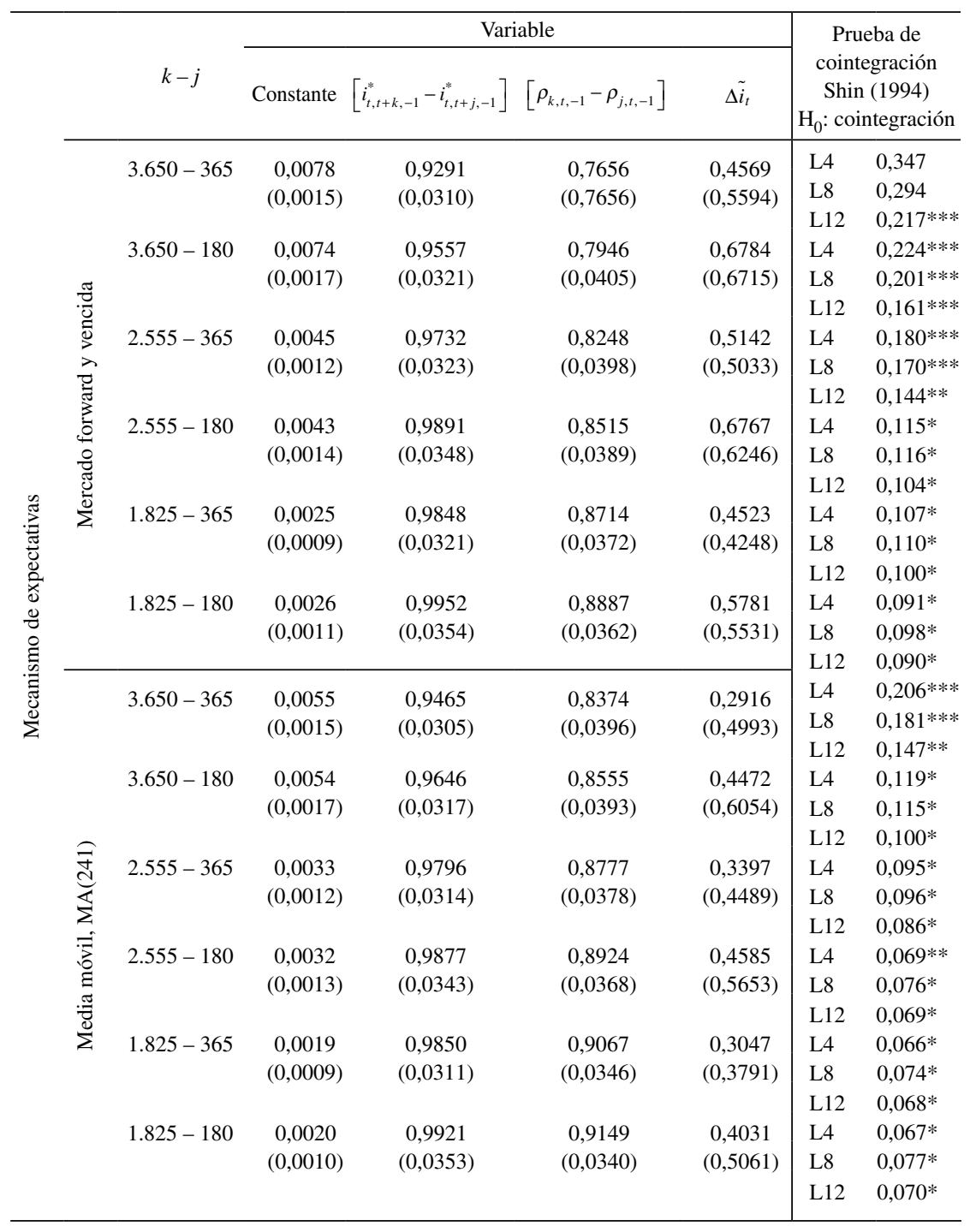

Nota: errores estándar entre paréntesis. * ** y *** se utilizan para denotar no rechazo de la hipótesis nula de la existencia de cointegración al $10 \%, 5 \%$ y $1 \%$, respectivamente, en la prueba de Shin (1994). 


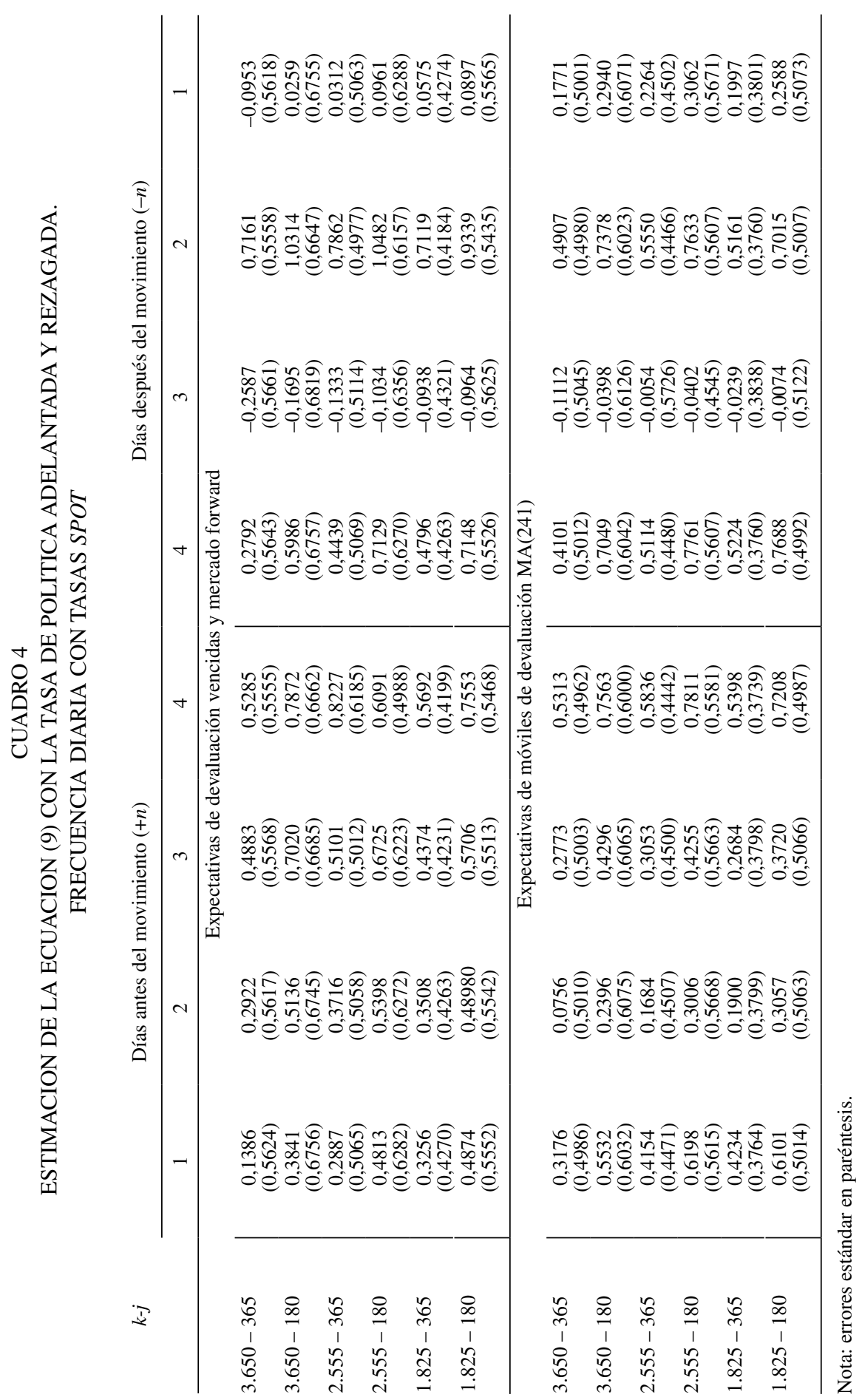


Los ejercicios de estimación que aparecen en los Cuadros 3 y 4 también fueron realizados utilizando la tasa forward, en lugar de la tasa spot, que se deriva de la curva cero cupón. Sin embargo, los resultados (no mostrados) no cambian: las intervenciones del BR no se traducen en ningún movimiento en las tasas de mercado a ninguno de los plazos considerados. Los demás resultados se mantienen. Se concluye, entonces, parcialmente, que las intervenciones del BR en el mercado de dinero a través de modificaciones a la tasa de expansión de subastas no tienen impactos estadísticamente significativos.

Como en las regresiones tipo $\mathrm{CH}$ analizadas en la sección anterior, la falta de validez empírica de nuestro enfoque puede tener varias explicaciones entre las que se cuentan: $i$ ) una alta credibilidad del BR durante el período de análisis que hace que las tasas del mercado no reaccionen en forma contemporánea ni hasta cuatro días antes o después de la intervención; ii) que no se cumple la hipótesis de expectativas (en lo que tiene que ver con los tramos intermedio y largo de la curva) y que en el corto plazo no hay ninguna conexión entre las tasas de política y las tasas del mercado secundario de deuda pública; o, iii) finalmente, que la frecuencia diaria no es la adecuada para verificar el impacto de los cambios de postura de política en las tasas de mercado. Para descartar esta última posibilidad, a continuación se realiza el ejercicio anterior utilizando los datos en frecuencia semanal, en cuyo caso las observaciones muestrales caen de 1.243 a 266.

\subsection{Resultados en frecuencia semanal}

Como se acaba de señalar, uno de los factores que puede estar impidiendo la observación de algún efecto de la variación en la tasa de política sobre las tasas de mercado de los títulos de deuda pública es la alta volatilidad que suelen exhibir los datos en frecuencia diaria. Por tal razón, se estima la ecuación (9) en frecuencia semanal tanto con la tasa de política del BR como con la tasa interbancaria (TIB), respectivamente, dada la evidencia de la estrecha asociación entre ambas (Huertas, et al., 2005 y Melo y Becerra, 2006). No obstante, los resultados sugieren que ni las variaciones de las tasas de política del BR (ver Cuadro 5) ni de la TIB (no mostrados por razones de espacio) afectan las tasas de mercado.

Los resultados presentados en el Cuadro 5 constituyen evidencia en contra de la hipótesis de que las variaciones en las tasas de política del BR afectan las tasas de retorno de los títulos de deuda pública y que, por tanto, son un canal verificado "del paso" de tasas de los préstamos a un día que realiza el BR a las tasas de mercado vistas a través de esta fracción del mismo. Estos resultados no sorprenden, a juzgar por la información que se desprende de la Figura 4. Sin embargo, cuando la ecuación (9) se estima adelantando y rezagando la tasa de política los resultados varían según se observa en el Cuadro 6. En estos casos se verifica la existencia de reacciones de las tasas de mercado entre 1 y 3 semanas antes del movimiento y tres semanas después del mismo. 


\section{CUADRO 5 \\ ESTIMACION DE LA ECUACION (9) EN FRECUENCIA SEMANAL CON TASAS SPOT}

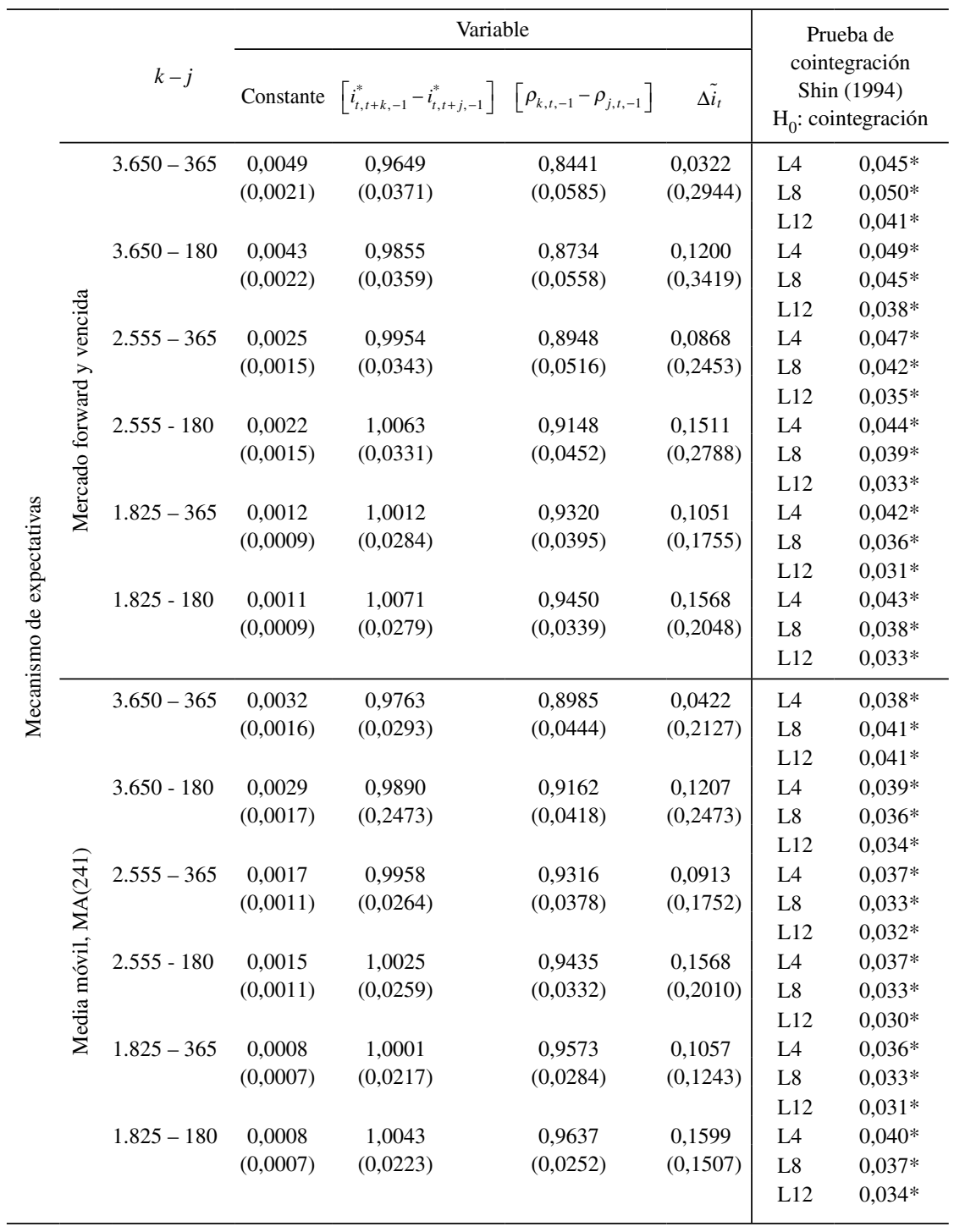

Nota: errores estándar entre paréntesis. *, ** y *** se utilizan para denotar no rechazo de la hipótesis nula de la existencia de cointegración al 10\%, 5\% y 1\%, respectivamente, en la prueba de Shin (1994). 


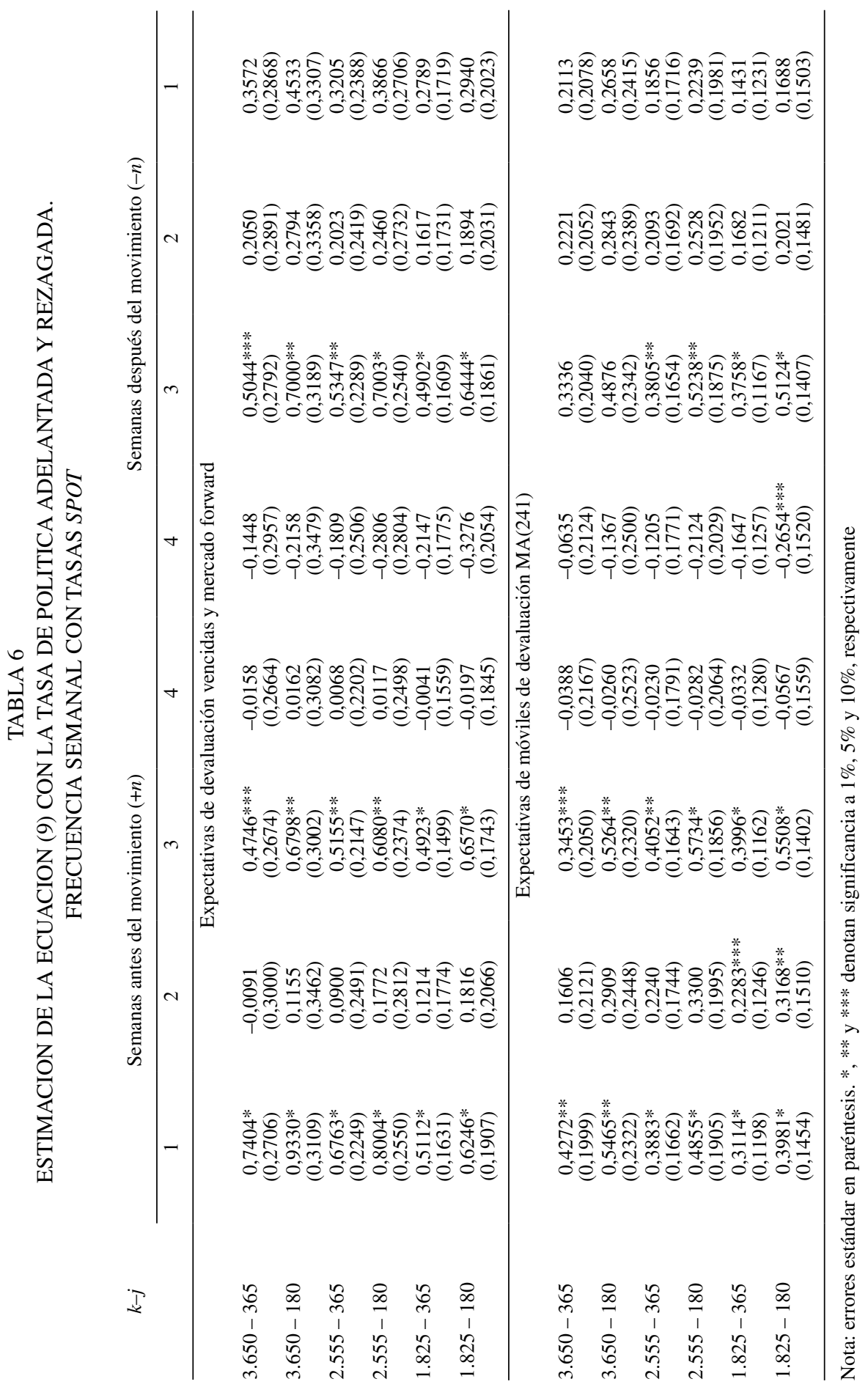


Es importante mencionar algunos puntos. En primer lugar, en los casos en los que los coeficientes asociados a las variaciones en las tasas de política son significativos, las variables están cointegradas. En segundo término, los coeficientes que resultaron significativos estadísticamente tienen el signo contrario al previsto por la teoría: en todos los casos son positivos para los dos períodos de estimación que se desprenden de los mecanismos de expectativas empleados. Este empinamiento $^{24}$ de la curva significa que la reacción de las tasas de mercado en forma anticipada o rezagada es mayor cuanto más lejano es el vencimiento de los títulos asociados a las mismas lo cual, de nuevo, va en contra de la hipótesis de expectativas. Esto se hace aún más evidente al analizar los coeficientes estimados. Por ejemplo, para el caso de los spreads 3.650-365 y 3.650-180 los coeficientes estimados para una semana antes del movimiento de tasas de política bajo los mecanismos de expectativas del mercado forward (o vencida), 0,7404 y 0,9330 respectivamente, sugieren que las reacciones son mayores para vencimientos a 365 días que para 180 días. De igual forma, sucede con los spreads 3.650-365 y 2.555-365, 0,7404 y 0,6763: es mayor la reacción del retorno de los títulos que vencen 3.650 días adelante que del retorno de los que vencen en 2.555 días. Todo lo anterior es contrario a la hipótesis de expectativas bajo credibilidad de la autoridad monetaria.

El Cuadro 7 contiene una información similar a la incluida en el Cuadro 6 pero las estimaciones se hicieron con la TIB en lugar de la tasa de política. Se observa que las tasas de mercado se mueven con claridad en todos los plazos. Esto debería ser un buen síntoma en cuanto a la habilidad del BR para transmitir de manera adecuada la intención que tiene en materia de tasas de interés dada la información macroeconómica de la que dispone y la probabilidad de alcanzar la meta de inflación. Sin embargo, el signo del coeficiente, de nuevo, es positivo lo cual sugiere un empinamiento de la estructura a plazo dejando muy poco espacio a la hipótesis de expectativas con credibilidad del BR.

La evidencia presentada en este documento sugiere que las intervenciones del BR pueden afectar la estructura a plazo dependiendo de la frecuencia en la que se observen los datos. En frecuencia diaria no parece haber ningún efecto ni contemporáneo, ni con algunos días de rezago o adelanto. En frecuencia semanal, es clara una reacción anticipada de los mercados entre una y tres semanas antes y alrededor de tres semanas después del movimiento en la tasa de política. Sin embargo, es muy probable que esta última se pueda estar confundiendo con el movimiento siguiente esperado en las tasas de política.

Independientemente de la frecuencia analizada, la evidencia sobre los comovimientos entre las tasas de interés locales y externas parece clara. En tal sentido, a movimientos en la curva spot externa el día anterior corresponden movimientos en la curva doméstica en el mismo sentido. Esto, de acuerdo con nuestra especificación, es síntoma del cumplimiento de la hipótesis de paridad descubierta ${ }^{25}$

\footnotetext{
${ }^{24}$ El fenómeno del empinamiento de la curva en lugar de un aplanamiento ya se había observado por algunos analistas locales (e.g., Porvenir, 2006).

${ }^{25}$ Cubierta cuando se trabaja con el tipo de cambio del mercado forward.
} 


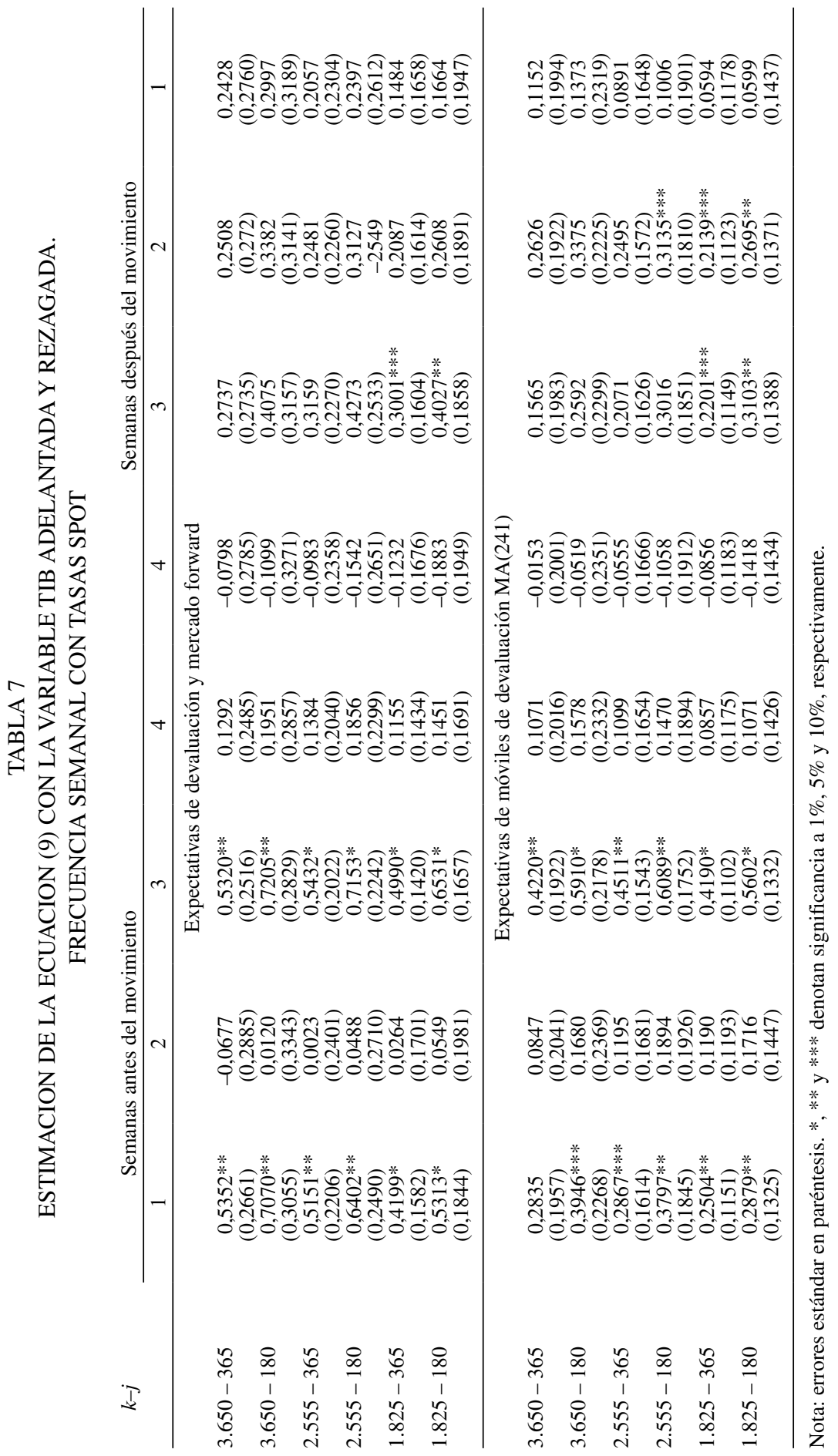


de intereses. De igual forma, diferencias en las primas de riesgo del día anterior se ven reflejadas en la estructura a plazo. Cuanto mayor sea la diferencia en las primas de riesgo entre los períodos $t+j$ y $t+k$ mayor será la tasa de largo plazo $(k)$ en relación con la de corto $(j)$ en el mercado secundario de deuda.

\section{CONCLuSIONeS}

Ampliando el enfoque original de Cook y Hahn (1989) con la hipótesis de paridad descubierta, de forma de considerar el efecto de los movimientos de capital explicado por los diferenciales de tasas de interés que afectan la composición de los portafolios, se verificó la hipótesis de que las variaciones de la tasa de subastas de expansión del Banco de la República afectan la estructura a plazo de las tasas de interés.

La muestra, en frecuencia diaria, comprende el período entre el 2 de enero de 2002 y el 31 de enero de 2007. Esto es equivalente a 266 semanas. Durante dicho período se registraron 18 movimientos en la tasa de expansión de subastas que es la tasa de política que ha venido utilizando el Banco.

Dadas las propiedades de estacionariedad de las series se usó un enfoque de cointegración (Phillips y Hansen, 1990). Se utilizaron tres especificaciones de las expectativas de devaluación. Con todas ellas se verifica el cumplimiento de la hipótesis de paridad descubierta de intereses. Esto es equivalente a señalar, que la estructura a plazo en Colombia se mueve por cambios en la curva de Estados Unidos el día anterior. Este es un hallazgo de la mayor importancia.

Los resultados en frecuencia diaria no muestran impacto ninguno en la estructura a plazo construida con base en el método de Nelson y Siegel (1987). Este resultado se mantiene no solo cuando se analiza de manera contemporánea sino también cuando se consideran hasta cuatro días antes y cuatro días después de la intervención del Banco de la República.

Con la utilización de los datos en frecuencia semanal tampoco se puede aceptar la hipótesis de que el Banco de la República puede alterar las tasas de retorno del mercado secundario de deuda de manera contemporánea. Este resultado, conjuntamente con los que se obtienen en frecuencia diaria, podría estar sugiriendo que la autoridad monetaria goza de alta credibilidad en el mercado, interpretación ésta que se vería reforzada por los resultados que se obtienen cuando las estimaciones se hacen considerando una, dos, tres o cuatro semanas antes o después de la adopción de la medida, puesto que allí sí se observan reacciones en el spread de tasas de interés domésticas. Es decir, se presenta evidencia de que el Banco de la República puede afectar las tasas de retorno del mercado secundario de deuda, ya que los agentes pueden anticipar las medidas que adoptará la autoridad. Por lo tanto, el Banco de la República sí tiene capacidad de afectar las expectativas inflacionarias y el valor de los activos de la economía colombiana.

Sin embargo, los signos de los coeficientes estimados son contrarios a los esperados de acuerdo con la hipótesis de expectativas racionales, ya que la curva se empina ante aumentos en la tasa de política. La curva se mueve una y tres semanas 
antes de la adopción de la medida pero con reacciones muy fuertes en los plazos largos en relación con los cortos. Al caer los precios relativos de los activos de largo plazo se prevén pérdidas de capital que motivan a los inversionistas a refugiarse en títulos de más corto plazo lo cual reafirma este resultado. De igual manera, los agentes se ven motivados a consumir más en el presente que en el futuro.

Roley y Sellon (1995) plantean que un empinamiento de la curva es posible cuando los agentes continúan esperando que la autoridad monetaria realice aumentos posteriores de la tasa de política. Esto a su vez es compatible con la interpretación de Romer y Romer (2000) si se cree que la autoridad monetaria fue sorprendida con nueva información sobre la trayectoria probable de la inflación futura. En cualquier caso, la posibilidad de tener una mayor inflación en el futuro no puede ser rechazada.

Una interpretación de los resultados es que es posible que el Banco de la República no haya sido lo suficientemente transparente (y coherente), como lo requiere el esquema de metas de inflación, para construir una credibilidad que le permita lograr reacciones en el mercado como las que sugieren los Diagramas 1 y 2. Una segunda posibilidad es que no se esté cumpliendo la hipótesis de expectativas de tasas de interés. Sin embargo, si este fuera el caso, la propia explicación podría estar en las medidas ejecutadas por la autoridad monetaria. Es posible que en el mercado de deuda exista un canal distinto al de expectativas como, por ejemplo, los que se desprenden de las teorías de preferencia por la liquidez o de hábitat preferidos.

En cualquier caso, dados el nivel de desarrollo de nuestro mercado, el conocimiento del esquema de metas de inflación, la estrategia de comunicación del Banco de la República y el tamaño muestral, los resultados de este trabajo son necesariamente provisionales y requieren la búsqueda de evidencia adicional que soporte o contradiga estos hallazgos.

De todas maneras, queremos plantear como tema de discusión la posibilidad de que los pronósticos del Banco de la República sobre inflación y las minutas de las deliberaciones que se realizan en el seno de la Junta Directiva del Banco de la República en materia de inflación sean entregados a los analistas del mercado ${ }^{26}$. El Banco de la República no debe abandonar el propósito de enviar señales claras al público no solo mediante los comunicados posteriores a las Juntas periódicas sino también mediante las acciones que adopta sobre distintas materias.

${ }^{26}$ Dos puntos que merecen especial atención son el período y los términos de su divulgación. 


\section{REFERENCIAS}

Amaya, C.A. (2006), "Interest Rate Setting and the Colombian Monetary Transmission Mechanism", Ensayos sobre Política Económica 50: 49-97.

Anderson, B. and J. Moore (1979), Optimal Filtering, Englewood Clis, NJ: Prentice Hall.

Arango, L.E., L.F. Melo y D. M. Vásquez (2003), "Estimación de la estructura a plazo de las tasas de interés en Colombia", Coyuntura Económica (Fedesarrollo) XXXIII, 1: 51-76.

Arango, L.E. y L.A. Flórez (2008), "Tramo corto de la curva de rendimientos, cambio de régimen inflacionario y expectativas de inflación en Colombia", El Trimestre Económico LXXV (1), 297: 183-210.

Ball, L. (1995), "Disinflation with imperfect credibility", Journal of Monetary Economics 35: 5-23.

Bank of England, Monetary Policy Committee (1999), The transmission mechanism of monetary policy. Bank of England, Quarterly Bulletin, May.

Barro, R. y R. Gordon (1983), "Rules, Discretion and Reputation in a Model of Monetary Policy", Journal of Monetary Economics, 17, 1: 101-122.

Betancourt, R., H. Vargas y N. Rodríguez (2006), "Interest rate pass-through in Colombia: a micro-banking perspective”, Borradores de Economía, No 407, Banco de la República.

BIS (2005), BIS Working Party on Monetary Policy in Latin America.

Blejer, M., A. Ize, A. Leone y S. Werlang (2000), Inflation Targeting in Practice strategic and Operational Issues and Application to Emerging Market Economies, International Monetary Fund.

Blinder, A.S. (2000), "Central-Bank Credibility: Why Do We Care? How Do We Build It?", American Economic Review, 90, 5: 1421-1431.

Boudoukh J., M. Richardson y R. Whitelaw (2005), “The Information in Long-Maturity Forward Rates: Implications for Exchange Rates and the Forward Premium Anomaly", NBER, Working Paper 11840.

Céspedes, L.F. y C. Soto (2005), "Credibility and inflation targeting in an emerging market: the case of Chile", Banco Central de Chile, Documentos de Trabajo № 312 .

Cook, T. y T. Hahn (1988), "The information of discount rate announcement and their effect on market interest rate", Journal of Money, Credit and Banking, 20, 2: 167-180.

Cook, T. y T. Hahn (1989), "The effect of Changes in the Federal Funds Rate Target on Market Interest Rates in the 1970s", Journal of Monetary Economics, 24: 331-351.

Cukierman, A. (1986), "Central Bank Behavior and Credibility: Some Recent Theoretical Developments", Federal Reserve Bank of St Louis Review, 68, 5: 5-17.

Dale, S. (1993), “The effect of Official Interest Rate Changes on Market Rates since 1987”, Bank of England, Working Paper Series $N^{\circ} 10$.

De Haan, J., S.C.W. Eijffinger y K. Rybinski, (2007), "Central Bank Transparency and Central Bank Communication: Editorial Introduction", European Journal of Political Economy, 23: 1-8.

Demiralp, S. y O. Jorda (2004), "The Response of Term Rates to Fed Announcements", Journal of Money, Credit and Banking, Vol. 36, № 3, 387-405.

Erceg, C.J. y A.T. Levin (2003), "Imperfect credibility and inflation persistence", Journal of Monetary Economics, 50, 915-944. 
Eijffinger S.C.W. y P. Geraats, 2006, "How transparent are central banks?", European Journal of Political Economy, 22: 1-21.

Escobar, J.F. y C.E. Posada (2004), "Dinero, precios, tasa de interés y actividad económica, un modelo del caso colombiano (1994:I - 2003:IV)", Borradores de Economía, No 303, Banco de la República.

Fernández, V. (2000), "Estructura de Tasas de Interés en Chile: ¿Qué tan Buen Predictor de Crecimiento e Inflación?", Cuadernos de Economía, Latin American Journal of Economics, año 37, 111: 373-404.

Gómez V. y A. Maravall (1994), "Estimation, prediction and interpolation for nonstationary time series with the Kalman filter", Journal of the American Statistical Association, 89: 611-624.

Gómez V. y A. Maravall (1996), "Programs TRAMO and SEATS, Instructions for the user", Working Paper $N^{\circ} 9628$, Banco de España.

Gómez V., A. Maravall y D. Peña (1999), "Missing observations in ARIMA models: skipping approach versus additive outlier approach", Journal of Econometrics, 88, 2: 341-363.

Geraats, P.M. (2002), “Central bank transparency”, Economic Journal, 112, 483: F532F565.

Haldane, A. y V. Read (1999), "Monetary policy and the yield curve, Bank of England", Quarterly Bulletin.

Haldane, A. y V. Read (2000), "Monetary policy surprises and the yield curve", Bank of England, Working Paper Series № 106.

Hamilton, J.D. (1994), Time Series Analysis, New Jersey, Princeton.

Huertas, C.A, M.A. Jalil, S. Olarte y J.V. Romero (2005), "Algunas consideraciones sobre el canal del crédito y la transmisión de las tasas de interés en Colombia", Borradores de Economía, № 351, Banco de la República.

Isard, P. (1996), "Uncovered Interest Parity", IMF Working Paper, 06/96.

Julio, J. (2001), "Relación entre la tasa de política del Banco de la República y las tasas del mercado: una exploración empírica", Borradores de Economía, № 188 , Banco de la República.

Kuttner, K. N. (2001), "Monetary policy surprises and interest rates: Evidence from the Fed funds futures market", Journal of Monetary Economics, 47, 3: 523-544.

Kydland, F.E. y E.C. Prescott (1977), "Rules Rather than Discretion: The inconsistency of Optimal Plans", Journal of Political Economy, 85, 3: 374-491.

Lyziak, T., J. Mackiewicz y E. Stanisławska (2007), "Central Bank Transparency and Credibility: the Case of Poland, 1998-2004", European Journal of Political Economy, 23: 67-87.

McCallum, B.T. (1994), "A reconsideration of the uncovered interest parity relationship", Journal of Monetary Economics, 33: 105-132.

Mehra, Y.P. (1994), “An Error-Correction Model of the Long-Term Bond Rate”, Federal Reserve Bank of Richmond Economic Quarterly, 80, 4: 49-68.

Melo, L.F. y O.R. Becerra (2006), "Una aproximación a la dinámica de las tasas de interés de corto plazo en Colombia a través de modelos GARCH multivariados", Borradores de Economía, № 366, Banco de la República.

Mishkin, S. F. (1995), "Symposium on the Monetary Transmission Mechanism", Journal of Economic Perspectives, 9, 4: 3-10.

Nelson, C.R. y A.F. Siegel (1987), "Parsimonious modelling of yield curves", Journal of Business, 60: 473-489. 
Phillips, P.C.B. y B. Hansen (1990), "Statistical Inference in Instrumental Variables Regression with I(1) Processes", Review of Economic Studies, 57: 99-125.

Porvenir (2006), "Inclinación en la curva de TES y la Tasa del Banco de la República", Gerencia de Investigaciones Económicas, Porvenir.

Roley, V. y G. Sellon (1995), "Monetary policy Actions and Long-Term Interest rates", Federal Reserve Bank of Kansas Economic Review, Q IV: 73-89.

Romer, C.D. y D.H. Romer, (2000), "Federal Reserve Information and the Behavior of Interest Rates", American Economic Review, 90, 3: 429-457.

Shin Y. (1994), "A residual-based test of the null of cointegration against the alternative of no cointegration", Econometric Theory, 10: 91-115. 


\section{ANEXO \\ El MÉtodo TRAMO DE INTERPolación}

La información disponible para la realización de los cálculos y estimaciones del trabajo es diaria y generada en su mayoría para los días hábiles del calendario colombiano. No obstante, en algunas ocasiones, debido, por un lado a los métodos de cálculo de las series o por otro, al escaso número de transacciones, no se lograban construir bases de datos completamente alineadas a nuestro calendario. Por tal motivo se interpolaron los datos faltantes, a fin de conseguir una base de datos que fuese equi-espaciada en concordancia a los días hábiles en Colombia. Este proceso de interpolación se realiza mediante el método TRAMO (Time series regression with Arima Noise, Missing observations and outliers).

TRAMO es un programa desarrollado por Gómez y Maravall (1994, 1996) y Gómez, Maravall y Peña (1998) utilizado para predecir, estimar e interpolar modelos de regresión con datos faltantes y errores de tipo ARIMA, en presencia de datos atípicos. El proceso de estimación permite el uso de variables exógenas como herramientas auxiliares para pronosticar valores faltantes. El modelo utilizado en este proceso se basa en las siguientes ecuaciones:

Sea $Z$ un vector de observaciones que puede incluir datos no observados:

(A1) $Z=\left(z_{1}, \ldots, z_{T}\right)$

Como etapa inicial se ajusta el siguiente modelo de regresión:

(A2) $Z_{t}=y_{t}{ }^{\prime} \beta+x_{t}$

Donde $\beta=\left(\beta_{1}, \ldots, \beta_{n}\right)^{\prime}$ es un vector de coeficientes de regresión, $y_{t}{ }^{\prime}=\left(y_{1 t}, \ldots y_{n t}\right)$ es un vector de $n$ variables exógenas y $x_{t}$ se asume como un proceso ARIMA, de manera que:

(A3) $\phi(B) \delta(B) x_{t}=\theta(B) a_{t}$

Siendo $B$ el operador de rezago; $\phi(B), \delta(B)$ y $\theta(B)$ polinomios finitos en $B$ y $a_{t} \sim$ i.i.d. $\left(0, \sigma_{a}^{2}\right)$.

El polinomio $\delta(B)$ contiene las raíces asociadas con la diferencia de la serie (tanto regulares como estacionales), $\phi(B)$ contiene las raíces estacionarias autorregresivas y $\theta(B)$ corresponde al polinomio de media móvil, el cual es invertible. $\phi(B)$ y $\theta(B)$ contienen polinomios asociados a comportamientos estacionales y no estacionales. Por lo tanto:

$$
\delta(B)=(1-B)^{d}\left(1-B^{s}\right)^{D}
$$




$$
\phi(B)=\left(1+\phi_{1} B+\ldots+\phi_{p} B^{p}\right)\left(1+\Phi_{1} B^{s}+\ldots+\Phi_{p} B^{s x P}\right)
$$

$$
\theta(B)=\left(1+\theta_{1} B+\ldots+\theta_{q} B^{q}\right)\left(1+\Theta_{1} B^{s}+\ldots+\Theta_{Q} B^{s x Q}\right)
$$

La estimación de los valores faltantes de $x_{t}$ se realiza con base en la información observada disponible. El interpolador o dato faltante se obtiene como el valor esperado de la serie en este período, dado el conjunto de información del resto de la muestra y corresponde al estimador que minimiza el error cuadrático medio.

La estimación de los datos faltantes es obtenida mediante estimaciones por máxima verosimilitud de procesos ARMA expresados en estado-espacio. El interpolador se obtiene a partir de algoritmos de suavización, tal como el suavizador de punto fijo de Anderson y Moore (1979).

La metodología TRAMO se aplica a las tasas spot de los TES denominados en pesos y calculadas a partir de las técnicas de splines cúbicos y Nelson y Siegel (1987) para todos los vencimientos considerados en este documento. El procedimiento TRAMO también se utiliza para el embi+ y para las tasas forward de los TES denominados en pesos y obtenidas a partir de splines cúbicos.

Como resultado de TRAMO se obtiene una serie de datos de periodicidad diaria equi-espaciada, esto quiere decir que se interpolaron aquellas fechas no correspondientes a sábados, domingos o festivos para las cuales no existían datos disponibles ${ }^{27}$. Este procedimiento se realiza para la muestra comprendida entre el 2 de enero de 2002 y el 31 de enero de 2007. En total se interpolaron 14 datos para las tasas y 23 para el embi+. Es importante señalar que en la interpolación de las tasas spot y forward se utilizan las intervenciones del Banco de la República como variable exógena.

${ }^{27}$ Para el caso de las tasas spot, este hecho puede derivarse de la inexistencia de operaciones, o que el número de operaciones realizados sea inferior al mínimo necesario para calcular la curva, para nuestro caso menos de 4 operaciones. 